\title{
Article \\ Combination Therapy of Novel Oncolytic Adenovirus with Anti-PD1 Resulted in Enhanced Anti-Cancer Effect in Syngeneic Immunocompetent Melanoma Mouse Model
}

\author{
Mariangela Garofalo ${ }^{1, *}\left(\mathbb{D}\right.$, Laura Bertinato ${ }^{1}$, Monika Staniszewska ${ }^{2}$, Magdalena Wieczorek ${ }^{3}(\mathbb{D}$, \\ Stefano Salmaso ${ }^{1} \mathbb{D}$, Silke Schrom ${ }^{4}$, Beate Rinner ${ }^{4}$, Katarzyna Wanda Pancer ${ }^{3} \mathbb{D}$ and Lukasz Kuryk ${ }^{3,5, * \mathbb{D}}$ \\ 1 Department of Pharmaceutical and Pharmacological Sciences, University of Padova, Via F. Marzolo 5, \\ 35131 Padova, Italy; laura.bertinato@studenti.unipd.it (L.B.); stefano.salmaso@unipd.it (S.S.) \\ 2 Centre for Advanced Materials and Technologies, Warsaw University of Technology, Poleczki 19, \\ 02-822 Warsaw, Poland; monika.staniszewska@pw.edu.pl \\ 3 Department of Virology, National Institute of Public Health-National Institute of Hygiene, Chocimska 24, \\ 00-791 Warsaw, Poland; mrechnio@pzh.gov.pl (M.W.); kpancer@pzh.gov.pl (K.W.P.) \\ 4 Division of Biomedical Research, Medical University of Graz, Roseggerweg 48, 8036 Graz, Austria; \\ silke.schrom@medunigraz.at (S.S.); beate.rinner@medunigraz.at (B.R.) \\ 5 Clinical Science, Targovax Oy, Lars Sonckin kaari 14, 02600 Espoo, Finland \\ * Correspondence: mariangela.garofalo@unipd.it (M.G.); lkuryk@pzh.gov.pl (L.K.)
}

Citation: Garofalo, M.; Bertinato, L.; Staniszewska, M.; Wieczorek, M.; Salmaso, S.; Schrom, S.; Rinner, B.; Pancer, K.W.; Kuryk, L. Combination Therapy of Novel Oncolytic Adenovirus with Anti-PD1 Resulted in Enhanced Anti-Cancer Effect in Syngeneic Immunocompetent Melanoma Mouse Model. Pharmaceutics 2021, 13, 547. https:// doi.org/10.3390/pharmaceutics 13040547

Academic Editor: Christopher Scott

Received: 26 March 2021

Accepted: 11 April 2021

Published: 14 April 2021

Publisher's Note: MDPI stays neutral with regard to jurisdictional claims in published maps and institutional affiliations.

Copyright: () 2021 by the authors. Licensee MDPI, Basel, Switzerland. This article is an open access article distributed under the terms and conditions of the Creative Commons Attribution (CC BY) license (https:// creativecommons.org/licenses/by/ $4.0 /)$.

\begin{abstract}
Malignant melanoma, an aggressive form of skin cancer, has a low five-year survival rate in patients with advanced disease. Immunotherapy represents a promising approach to improve survival rates among patients at advanced stage. Herein, the aim of the study was to design and produce, by using engineering tools, a novel oncolytic adenovirus AdV-D24- inducible co-stimulator ligand (ICOSL)-CD40L expressing potent co-stimulatory molecules enhancing clinical efficacy through the modulation of anti-cancer immune responses. Firstly, we demonstrated the vector's identity and genetic stability by restriction enzyme assay and sequencing, then, by performing in vitro and in vivo pre-clinical studies we explored the anti-cancer efficacy of the virus alone or in combination with anti PD-1 inhibitor in human melanoma cell lines, i.e., MUG Mel-1 and MUG Mel-2, and in immunocompetent C57BL/6 melanoma B16V mouse model. We showed that both monotherapy and combination approaches exhibit enhanced anti-cancer ability and immunogenic cell death in in vitro settings. Furthermore, AdV-D24-ICOSL-CD40L combined with anti PD-1 revealed a fall in tumor volume and $100 \%$ survival in in vivo context, thus suggesting enhanced efficacy and survival via complementary anti-cancer properties of those agents in melanoma therapy. Collectively, the novel oncolytic vector AdV-D24-ICOSL-CD40L alone or in combination with anticancer drugs, such as check point inhibitors, may open novel therapeutic perspectives for the treatment of melanoma.
\end{abstract}

Keywords: melanoma; immunotherapy; oncolytic adenovirus; PD-1 inhibitors; combinatory therapy; ICOSL; CD40L

\section{Introduction}

Malignant melanoma is considered to be the most dangerous type of skin cancer that develops from the melanocytes, the pigment-producing cells [1-3]. In most cases, melanoma occurs in the skin [2], however, it can also appear in the mouth, intestines, and in the eye (known as uveal melanoma) [1,2]. Around the world, as of 2012, about 232,000 people were newly diagnosed with melanoma. The highest numbers of melanoma cases occur in New Zealand (the overall annual age-standardized rate (ASR) is 35.8), Australia (ASR = 34.9) [4-6], the northern part of Europe (ASR = 14.6) and in North America (ASR = 13.8); however, in Asia (ASR = 1.7), Africa (ASR = 1.1), and South America $(\mathrm{ASR}=2.5)$ cases are comparatively less [4,5]. Additionally, men are more commonly affected by this disease compared to women (1.6 times) $[1,2,6]$. 
The rise of immune checkpoint inhibitors (ICIs) has significantly improved the treatment paradigm for melanoma [7]. The recent advances have been achieved due to adoption of antibodies against the immune checkpoints: programmed cell death protein-1 (anti-PD-1, pembrolizumab, nivolumab), cytotoxic T-lymphocyte-associated protein-4 (anti-CTLA-4, ipilimumab) and programmed death-ligand 1 (anti-PD-L1, atezolizumab, durvalumab, avelumab) $[7,8]$. These ICIs have shown profound clinical efficacy in metastatic melanoma by reversing effector T-cell exhaustion and dysfunction, leading to the improvement of their anti-tumoral properties, thereby increasing T-cell activation [9]. However, unfortunately, from $40 \%-60 \%$ of melanoma patients do not gain any significant therapeutic benefit and a profound proportion of responders observe tumor relapse within 2 years [9-11]. The response rates to ICI monotherapy are low, in part due to the presence of a "cold" immune tumor microenvironment (TME) [12]. Consequently, novel and more efficient therapeutics strategies and synergistic combination therapies are in high demand $[9,12]$.

The fast-accelerating oncolytic virotherapy is a promising anti-cancer strategy. Oncolytic viruses are naturally occurring or genetically modified viruses that infect, replicate in, and kill cancer cells without harming normal cells [13-15]. Significant advances in gene engineering capabilities and refinements in vector production have been made within the last few decades [16-18], especially because of a better understanding of how oncolytic viruses modulate the TME, which has raised the field "oncolytic immunotherapy". Oncolytic adenoviruses after their injection to the tumor lesion lead to increased levels of proinflammatory cytokines and an influx of NK cells, T cells, and antigen-presenting cells (APC) $[7,19]$. Notably, PD-L1 expression is known to elevate on cancer cells and immune cells following viral infection $[20,21]$. Taken together, these cascades or reactions modulate the TME and reverse it from "cold" to "hot" with a development of cytokines and immune effectors. Talimogene laherparepvec (T-VEC; $\operatorname{Imlygic}^{\mathrm{TM}}$ ), is a genetically engineered herpes simplex virus, type 1 , and is the first oncolytic virus therapy to be approved for the treatment of advanced melanoma by the US FDA. T-VEC showed improvement in durable response rate (DRR), objective response rate (ORR), and progression-free survival (PFS) in a randomized phase III clinical trial for patients with advanced melanoma [22].

It has been shown that ICIs work better in the presence of lymphocytic infiltrate, which is not always the case in many cancer types, including melanoma $[20,23]$. Oncolytic viruses (OVs) induce anti-cancer immune responses that enhance the efficacy of checkpoint inhibitors. For this reason, the combinatory therapy of oncolytic vectors with ICI is considered to be a promising regime in melanoma therapy. Furthermore, the efficacy of combining OVs and ICIs has been shown in pre-clinical findings, and there are currently many ongoing clinical trials testing combination therapies with promising results with ICIs $[24,25]$.

Nevertheless, despite extensive research, oncolytic virotherapy has shown limited efficacy against solid tumors as monotherapy $[26,27]$. Therefore, the development of novel and more potent oncolytic vectors is highly needed. Additionally, combinatory therapies are considered to be the future direction in cancer treatment. In fact, T-VEC demonstrated improved survival outcomes for unresectable melanoma in combination [28].

In the present preclinical study, we engineered a novel oncolytic vector, expressing two potent co-stimulatory ligands aiming at enhancing anti-cancer immune responses by $\mathrm{T}$ cell co-activation. Therefore, in this study we investigated whether the targeted delivery of co-stimulatory proteins mediated by OVs may improve anti-cancer immune responses to the melanomas and reduce any potential undesired off-target effects. To this aim, the novel oncolytic adenovirus AdV-D24- inducible co-stimulator ligand (ICOSL)-CD40L was designed and produced to selectively replicate in cancer but not in healthy cells. Indeed, the virus antitumor properties will result in the eradication of cancer cells and, additionally, the production of exogenous proteins that modulate anti-cancer activity through distinct mechanisms that can synergize with other anticancer agents. The OV has been armed with two potent co-stimulatory molecules: inducible co-stimulator ligand (ICOSL) and CD40 ligand (CD40L, CD154). Inducible co-stimulator (ICOS) is a CD28-related molecule 
expressed on activated T cells, able to interact with its ICOSL present on APCs such as dendritic cells (DCs), B lymphocytes, and some cancer cells [29,30]. Furthermore, ICOSL expression in solid tumors support the activation of CD8+ cytotoxic T cells, thus inducing anti-tumor immune responses [31,32]. Remarkably, it has been also reported that ICOSL transfected tumor cells showed that the ligand positively supports the tumor regression through the activation of CD8+ cytotoxic T-mediated pathways [29,33]. Indeed, Zamarin et al. cloned a recombinant Newcastle disease virus expressing ICOS ligand (NDV-ICOSL) and showed that the injection of NDV-ICOSL in vivo resulted in improved infiltration of activated $\mathrm{T}$ cells in both virus-injected and non-injected tumors, leading to the rejection of both tumors when used in combination with CTLA-4 inhibitor [34]. Additionally, it has been also found that CD40 is expressed on B cells, macrophages and DCs and the interaction of CD40-CD40L leads to the activation of adaptive immune responses, including the development of CD8+ cytotoxic T lymphocytes (CTLs) [35]. Moreover, previous studies by Diaconu et al. reported that oncolytic adenovirus coding for CD40L significantly inhibited tumor growth in vivo by exhibiting both oncolytic and apoptotic effects, thus resulting in enhanced calreticulin exposure and high-mobility group box 1 (HMGB1) and ATP release [36]. Taking together these considerations, the presence of both ICOSL and CD40L, which are expressed locally in tumor microenvironment by the vector, should boost the anti-tumor immune responses.

Herein, by exploring the antitumor activity of a novel oncolytic adenovirus AdV-D24ICOSL-CD40L in combination with anti PD-1 in in vitro and in vivo in immunocompetent mouse model engrafted with murine melanoma cells, we demonstrated that a therapy with OVs expressing potent immune modulators can be an effective strategy to drive the systemic efficacy of an immune checkpoint blockade. Our in vitro and in vivo preclinical findings support the notation that such combination therapy may enhance anti-cancer efficacy and survival via targeted cancer cell lysis and the induction of immunogenic cell death. The outcome of this study may provide important insights to further study the combination therapy approaches using oncolytic adenoviruses and ICIs in solid tumors. Clearly, future investigations will assess the role of ICOSL and CD40L in tumor cells and support possible therapeutic recommendations for the management and treatment of melanoma patients.

\section{Materials and Methods}

\subsection{Cell Lines, Anti-PD1 Antibodies}

Human melanoma cell lines, MUG Mel-1, derived from human brain metastasis, MUG Mel-2 derived from a skin lesion and B16V mouse melanoma cells were kindly provided by Prof. Rinner from the Medical University of Graz and were cultured in RPMI 1640 media (Gibco Laboratories, CA, USA) supplemented with 1\% of penicillin/streptomycin (Gibco Laboratories) and 1\% L-glutamine (Gibco Laboratories) and 10\% fetal bovine serum (FBS, Gibco Laboratories, USA). A549 cells were ordered from the American Type Culture Collection (ATCC, USA) and cultured at $37^{\circ} \mathrm{C}, 5 \% \mathrm{CO}_{2}$ in DMEM (Lonza, Switzerland) supplemented with 10\% FBS (Gibco Laboratories, USA), $1 \%$ of $100 \mathrm{U} / \mathrm{mL}$ penicillin/streptomycin (Gibco Laboratories) and 1\% L-glutamine (Gibco Laboratories). Pembrolizumab (Keytruda, MSD) and purified anti-mouse CD279 (PD-1) antibody have been resuspended according to manufacturers' instructions (BioLegend).

\subsection{Generation and Production of Oncolytic Adenoviruses}

The oncolytic virus used in this work was the adenovirus AdV-D24-ICOSL-CD40L, a chimeric serotype 5/3 adenovirus, with a 24-bp deletion in E1A gene and a chimeric fiber knob domain (hybrid 5/3 serotype) and AdV-D24, a vector without co-stimulatory molecules and owning a similar backbone.

The vector AdV-D24-ICOSL-CD40L was designed by authors and purchased (AdenoQuick Kit, OD260 Inc., Boise, OH, USA). AdV-D24 was generated and amplified using adenovirus preparation techniques [37]. The shuttle plasmid containing genes of interest 
(D24, fiber modifications 5/3, ICOSL + CD40L) were constructed. Subsequently, the cosmid was engineered by the digestion of the shuttle plasmids with SfiI and ligating them to each other. The ligation products were packed into phage lambda, infected Escherichia coli, and selected of ampicillin and kanamycin-resistant clones. The identity of obtained colonies was confirmed by restriction analysis or sequencing. Finally, DNA transfection was made in order to rescue the virus by the linearization of the cosmid with PacI or SwaI enzymes. The linear cosmid was transfected into helper cells (human lung cancer cells: A549) and plaques harvested. The virus DNA sequence/genome integrity was confirmed by restriction analysis or sequencing.

Oncolytic adenovirus was characterized by performing titration $(\mathrm{VP} / \mathrm{mL})$, expanded in A549 cells and purified on cesium chloride gradients. The OD-260-SDS method was used to determine the concentration of virus particles in the purified stock of viruses $(\mathrm{VP} / \mathrm{mL})$. The concentration of virus particles (VPs) in the preparations was calculated given the extinction coefficient of $1.1 \times 10^{12}$ virus particles (VPs) per Abs260 unit in the presence of SDS. The OD-260-reading is a simple, accurate, and precise method to determine adenovirus particle concentration using OD260 $\mathrm{nm}$ absorbance. The method guarantees the complete disruption of virus particles and viral DNA prior to absorbance measurements, therefore eliminating absorbance measurement errors. The application of this modified method should reduce interlaboratory variability in determining adenovirus particle concentrations [38]. This method is commonly and widely used in pre-clinical and clinical studies for a dose determination worldwide. The TCID50 assay was also performed to quantify viral titers by determining the concentration at which $50 \%$ of the infected cells display cytopathic effect (CPE, IU/mL)). Nevertheless, due to the high variability of this assay, it was not used in downstream analyses. The ratio between VP/IU was also calculated (VP/mL/IU/mL). and ranged from 11 to 13 and 21 (mean 1:17), and was comparable between tested stocks (ratio $\mathrm{VP} / \mathrm{mL} / \mathrm{IU} / \mathrm{mL}$ ).

\subsection{Restriction Enzyme Assay (REA)}

Viral DNA was extracted from Ad5-D24-ICOSL-CD40L infected A549 cells according to the Hirt method $[39,40]$. The identity of the virus was assessed by restriction digestion with BamHI and NdeI. All restriction patterns of AdV-D24-ICOSL-CD40L \#1-2-3-4 viral DNA match that of the PacI-digested cosmid pAdV-D24-ICOSL-CD40L, indicating the stability of the vector. The presence of the ICOSL-CD40L cassette in the vector was confirmed by the presence of the restriction fragments, highlighted in green in the pictures below.

\subsection{Whole Genome Sequencing}

Viral DNA was extracted from $\mathrm{CsCl}$-purified virus particles using proteinase $\mathrm{K}$. AdVD24-ICOSL-CD40L sequencing library was made using a Nextera XT kit (Illumina). The size of the library was assessed by high-resolution agarose gel electrophoresis. In order to assess the consensus sequence, the AdV-D24-ICOSL-CD40L library was sequenced on an iSEQ100 instrument (Illumina). The $>4.7 \mathrm{~m}$ reads (FastQ files) were assembled into a single contig using the program BWA Aligner and the predicted sequence of the AdV-D24ICOSL-CD40L genome as reference. The contig was visualized using the software Geneious v11.1.5 (Biomatters Ltd., Auckland, New Zealand). The contig calculated a 36,478 bp-long consensus sequence, with a sequence coverage ranging between 911 and $\sim 31,000$. In order to derive the sequence alignment, the program SnapGene v5.0.7 was used to align the consensus sequence to the predicted sequence of the virus genome. The alignment confirmed the integrity of the entire genome of AdV-D24-ICOSL-CD40L, including the 24-bp deletion in the E1A CR2 domain, the CMV-ICOSL-IRES-CD40L expression cassette inserted in place of the E3 region and the hybrid Ad5/3 fiber. The presence of the 24-bp deletion in the E1A CR2 domain, the CMV-ICOSL-IRES-CD40L expression cassette inserted in place of the E3 region and the hybrid Ad5/3 fiber were confirmed. Analysis of sequence coverage did not detect the presence of a virus sub-population with a genome characterized by a major rearrangement such as a large insertion or deletion. 


\subsection{Western Blot}

The expression of ICOSL and CD40L from AdV-D24-ICOSL-CD40L was assessed by infecting A549 cells with the virus (A549 cells were seeded in 6-cm dishes and were infected with $0.5 \mathrm{~mL}$ of crude lysate of viral clones \#1-2-3-4) and harvesting proteins $48 \mathrm{~h}$ after the infection and detecting ICOSL and CD40L by Western blot. Infected cell pellets were washed twice with cold PBS, then lysed by the addition of ice-cold NP40 cell lysis buffer (Invitrogen \#FNN0021). After 30 min incubation at $4{ }^{\circ} \mathrm{C}$ with gentle shaking, the cell lysates were clarified by centrifugation (4000 rpm). The protein concentration was measured using a BCA assay (Pierce). CD40L was detected using the primary antibody: goat polyclonal anti-CD40L antibody (R\&D \#AF617), followed by the secondary antibody: rabbit anti-goat IgG (HRP-conjugated, R\&D HAF017). In turn, the ICOSL was detected by staining with primary antibody: mouse monoclonal anti-ICOS ligand antibody (Novus Biologicals \# NPB2-46011) and subsequently with the secondary antibody: donkey anti-mouse IgG (HRPconjugated, R\&D HAF018). HRP detection was performed with a SuperSignal West Pico Chemiluminescent substrate (Pierce \#34080) and visualized with a Kodak 440CF imager.

\subsection{CAR and DSG2 Expression in Melanoma Cell Lines}

MUG Mel- 1 and MUG Mel- 2 cells were seeded at the concentration of $5 \times 10^{5}$ cell/well and maintained under the above standard cell culture growth conditions. On the next day, melanoma cells were stained firstly with mouse monoclonal anti-CAR antibody (Santa Cruz Biotech, Dallas, TX, USA) and then with 1:2000 Alexa-Fluor 488 secondary antibody (Abcam, Cambridge, UK) or mouse monoclonal anti-DSG2 antibody (Abcam, Cambridge, UK) and then with 1:2000 Alexa-Fluor 488 secondary (Beckman-Coulter Cytomics FC500).

\subsection{Cell Viability: MTS Cytotoxicity Assay}

Human melanoma cells MUG Mel-1, MUG Mel-2 cells and murine melanoma B16V were seeded at a density of $1 \times 10^{4}$ cells/well in a 96-well plate and maintained under standard growth condition. After overnight incubation, cells were treated as follows: (i) AdV-D24 (0.1, 1, 10, 100 VP/cell), (ii) AdV-D24-ICOSL-CD40L (0.1, 1, 10, 100 VP/cell), (iii)

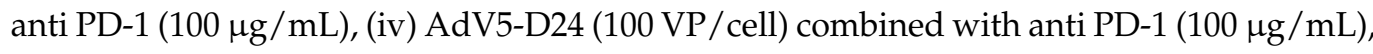
(v) Ad5V-D24-ICOSL-CD40L (100 VP/cell) combined with anti PD-1 $(100 \mu \mathrm{g} / \mathrm{mL})$. Cell viability was determined $96 \mathrm{~h}$ after treatment, by using CellTiter 96 AQueous One Solution Cell Proliferation Assay (MTS) according to the manufacturer's instructions (Promega, Madison, WI, USA). The absorbance was measured with a 96-well plate spectrophotometer (Victor Nivo $^{\mathrm{TM}}$, PerkinElmer, Milano, Italy) at $490 \mathrm{~nm}$. The experiments were independently performed three times and each treatment was performed in triplicate.

\subsection{Immunogenicity of Tumor Cell Death In Vitro and Ex Vivo}

Calreticulin (CRT) exposure: Human and murine melanoma cell lines were seeded in triplicate onto 24-well plates at a concentration of $5 \times 10^{4}$ cells/well and maintained under standard growth condition. On the following day, cells were treated as follows: (i) AdV-D24 (100 VP/cell), (ii) AdV-D24-ICOSL-CD40L (100 VP/cell), (iii) anti PD-1 (100 $\mu \mathrm{g} / \mathrm{mL})$, (iv) AdV5-D24 (100 VP/cell) combined with anti PD-1 (100 $\mu \mathrm{g} / \mathrm{mL})$, (v) Ad5V-D24-ICOSLCD40L (100 VP/cell) combined with anti PD-1 $(100 \mu \mathrm{g} / \mathrm{mL})$. Then, $48 \mathrm{~h}$ after treatment, cells were harvested and stained with 1:1000 diluted Alexa-Fluor 488 rabbit polyclonal anti-calreticulin antibody (Abcam, Cambridge, UK) (concentration of $1 \mu \mathrm{g} / \mathrm{mL}$ ) or Alexa Fluor Plus 488 goat anti-mouse at the concentration 1-10 $\mu \mathrm{g} / \mathrm{mL}$ (ThermoFisher, Scientific, A32723, Waltham, MA, USA) for $30 \mathrm{~min}$ and analyzed by flow cytometry analysis using Beckman-Coulter Cytomics FC500. The experiments were independently performed three times and each treatment was performed in replicates. Additionally, resected tumor tissue from all mice groups (described under the Section 2.10) were collected and processed at sacrifice. A uniform single-cell suspension from tissues were obtained using cell strainers (Corning, $100 \mu \mathrm{m}$ ). Subsequently, the single cell suspension was used for the detection of 
CRT exposure on the cancer cells surface. Cells were harvested and stained by following the same staining protocol as described for the in vitro part above.

ATP release: Human and murine melanoma cells were seeded at a concentration of $1 \times 10^{4}$ cells/well in 96-well plates. On the following day, cells were treated as follows: (i) AdV-D24 (100 VP/cell), (ii) AdV-D24-ICOSL-CD40L (100 VP/cell), (iii) anti PD-1 $(100 \mu \mathrm{g} / \mathrm{mL})$, (iv) AdV5-D24 (100 VP/cell) combined with anti PD-1 (100 $\mu \mathrm{g} / \mathrm{mL}),(\mathrm{v})$ Ad5V-D24-ICOSL-CD40L (100 VP/cell) combined with anti PD-1 $(100 \mu \mathrm{g} / \mathrm{mL})$. Supernatants were collected after $72 \mathrm{~h}$ and analyzed with an ATP detection kit (CellTiter-Glo ${ }^{\circledR}$ Luminescent Cell Viability Assay, Promega) according to the manufacturer's protocol for luminometric analysis (Victor $\mathrm{Nivo}^{\mathrm{TM}}$ ). The experiments were independently performed three times and each treatment was performed in replicates. Additionally, resected tumor tissue from all mice groups (described under the Section 2.10) were collected and processed at sacrifice. A cell suspension from tissues were obtained using cell strainers (Corning, $100 \mu \mathrm{m})$. Subsequently, the cell supernatant was used for the detection of ATP release from the cancer cells. Supernatants were collected and analyzed with an ATP detection kit as described for the in vitro part above.

HGMB-1 release: Human melanoma cells were seeded at density of $1 \times 10^{4}$ cells per well in 96-well plate and maintained under standard growth condition. On the following day, cells were treated as follows: (i) AdV-D24 (100 VP/cell), (ii) AdV-D24-ICOSL-CD40L (100 VP/cell), (iii) anti PD-1 (100 $\mathrm{\mu g} / \mathrm{mL})$, (iv) AdV5-D24 (100 VP/cell) combined with anti PD-1 (100 $\mu \mathrm{g} / \mathrm{mL})$, (v) Ad5V-D24-ICOSL-CD40L (100 VP/cell) combined with anti PD-1 $(100 \mu \mathrm{g} / \mathrm{mL})$. Supernatants were collected after $72 \mathrm{~h}$ and HGMB-1 levels were detected with an Elisa assay kit (MBL International, Woburn, MA, USA), following manufacturer's instruction.

\subsection{Evaluation of the Concentration of the ICOSL and CD40L Produced by the Virus}

MUG Mel-1 and MUG Mel-2 cells were seeded at $1 \times 10^{5}$ cells $/ \mathrm{mL}$ in a 96-well plate and maintained under standard growth condition. After overnight incubation, cells were treated as described above. Supernatants were collected $72 \mathrm{~h}$ after treatments and analyzed for human ICOSL and CD40L concentration using ELISA kits (LifeSpan BioSciences, Inc, Seattle, WA, USA, LS-F9059, RayBiotech, ELH-CD40L-1, Peachtree Corners, GA, USA) according to the manufacturer's instructions.

\subsection{In Vivo Efficacy Studies}

All animal procedures were performed and approved by the Austrian Federal Ministry of Science and Research (BMWF) (GZ 66.010/0058-V/3b/2019) and Italian Ministry of Health (117/2020-PR). For the efficacy experiment, murine xenografts were established by subcutaneously (s.c.) injecting $1 \times 10^{6} \mathrm{~B} 16 \mathrm{~V}$ melanoma cells into both flanks of 10-week-old C57BL/6 male mice (6 tumors/group). Tumors (two tumors per mouse, $\sim 5 \times 5 \mathrm{~mm}$ in diameter) and were randomized prior the treatment initiation as follows: vehicle (100 $\mu \mathrm{L}$ of PBS) administered intratumorally (i.t.), AdV-D24 (administered i.t at a concentration of $1.75 \times 10^{10} \mathrm{VP} /$ tumor $\left(3.5 \times 10^{10} \mathrm{VP} / \mathrm{mouse}\right)$, AdV-D24-ICOSL-CD40L administered i.t. at a concentration of $1.75 \times 10^{10} \mathrm{VP} /$ tumor $\left(3.5 \times 10^{10} \mathrm{VP} /\right.$ mouse), murine anti-PD1 (purified anti-mouse CD279 (PD-1) antibody BioLegend) administered intravenously (i.v.) $(200 \mu \mathrm{g} / \mathrm{mouse})$, AdV-D24 (administered i.t. at a concentration of $1.75 \times 10^{10} \mathrm{VP} /$ tumor $\left(3.5 \times 10^{10} \mathrm{VP} /\right.$ mouse $)$ followed by i.v. treatment with anti-PD1 (200 $\mu \mathrm{g} / \mathrm{mouse})$, AdV-D24-ICOSL-CD40L administered i.t. at a concentration of $1.75 \times 10^{10} \mathrm{VP} /$ tumor $\left(3.5 \times 10^{10} \mathrm{VP} /\right.$ mouse $)$ followed by i.v. treatment with anti-PD1 (200 $\mu \mathrm{g} /$ mouse) (Table 1). Tumor/lesion size was recorded using caliper on two dimensions every three days. The longest and shortest diameter of tumor at each timepoint were recorded and the tumor volume was calculated using a formula of $0.52 \times$ length 3 (width) ${ }^{2}$. All animals were observed for clinical signs, morbidity or mortality daily during the acclimatization and administration period and additionally $30 \mathrm{~min}$ after each treatment. 
Table 1. Overview of the treatment scheme. i.t: intratumorally; i.v: intravenously; ICOSL: inducible co-stimulator ligand.

\begin{tabular}{|c|c|c|c|c|c|c|c|}
\hline Group & Tumor Cell Engraftment & Day 1 & Day 2 & Day 3 & Day 4 & Day 5 & Day 6 \\
\hline $\begin{array}{l}\text { 1. Mock } \\
\text { 2. AdV-D24-ICOS-CD40L } \\
\text { 3. AdV-D24-WT } \\
\text { 4. Anti PD-1 } \\
\text { 5. AdV-D24-ICOS-CD40L + } \\
\text { anti PD-1 } \\
\text { 6. AdV-D24-WT + anti } \\
\text { PD-1 }\end{array}$ & $\begin{array}{c}\text { Tumor inoculation: } \\
2 \text { tumors per mouse, one } \\
\text { tumor } / \text { flank. } \\
\text { Each flank was engrafted } \\
\text { with } 1 \times 10^{6} \mathrm{~B} 16 \mathrm{~V} \text { cells in } \\
50-100 \mu \mathrm{L} \\
\text { (6 tumors per group) }\end{array}$ & $\begin{array}{l}\text { PBS i.t. } \\
\text { PBS i.v. } \\
\text { Virus i.t. } \\
\text { Virus i.t. } \\
200 \mu \text { i.v. } \\
\text { Virus i.t. + } \\
200 \mu \text { g i.v. } \\
\text { Virus i.t. + } \\
200 \mu \text { g i.v. }\end{array}$ & $\begin{array}{l}\text { PBS i.t. } \\
\text { PBS i.v. } \\
\text { Virus i.t. } \\
\text { Virus i.t. } \\
200 \mu \text { i.v. } \\
\text { Virus i.t. + } \\
200 \mu \text { i.v. } \\
\text { Virus i.t. + } \\
200 \mu \text { i.v. }\end{array}$ & $\begin{array}{l}\text { PBS i.t. } \\
\text { PBS i.v. } \\
\text { Virus i.t. } \\
\text { Virus i.t. } \\
200 \mu \text { i.v. } \\
\text { Virus i.t. + } \\
200 \mu \text { i.v. } \\
\text { Virus i.t. + } \\
200 \mu \text { g i.v. }\end{array}$ & $\begin{array}{l}\text { PBS i.t. } \\
\text { PBS i.v. } \\
\text { Virus i.t. } \\
\text { Virus i.t. } \\
200 \mu \text { i.v. } \\
\text { Virus i.t. + } \\
200 \mu \text { i.v. } \\
\text { Virus i.t. + } \\
200 \mu \text { i.v. }\end{array}$ & $\begin{array}{l}\text { PBS i.t. } \\
\text { PBS i.v. } \\
\text { Virus i.t. } \\
\text { Virus i.t. } \\
200 \mu \text { i.v. } \\
\text { Virus i.t. + } \\
200 \mu \text { i.v. } \\
\text { Virus i.t. + } \\
200 \mu \text { g i.v. }\end{array}$ & $\begin{array}{l}\text { PBS i.t. } \\
\text { PBS i.v. } \\
\text { Virus i.t. } \\
\text { Virus i.t. } \\
200 \mu g \text { i.v. } \\
\text { Virus i.t. + } \\
200 \mu \text { i.v. } \\
\text { Virus i.t. + } \\
200 \mu \text { i.v. }\end{array}$ \\
\hline
\end{tabular}

\subsection{Statistical Analysis}

Data were reported as mean \pm SEM or as indicated. Statistical analysis was performed with GraphPad Prism software version 8 (La Jolla, San Diego, CA, USA). An un-paired test and one-way ANOVA with the Krusal-Wallis test was used to compare two or more groups. Survival curves and their statistical analysis were performed using the Kaplan-Meier test.

\section{Results}

\subsection{Cloning, Characterization, Confirmation of Genetic Stability and Identity of the Double Transgene Vector Expressing Co-Stimulatory Transgenes (ICOSL and CD40L)}

Since the treatment with oncolytic viruses is a promising approach for targeting the immune response against the tumor by combining the cytolytic activity together with the ability of the viruses to activate the immune system [41,42], in the current study, we aimed to design and engineer a novel chimeric oncolytic adenovirus vector AdVD24-ICOSL-CD40L armed with two potent co-stimulatory molecules, ICOSL and CD40L, selectively expressing in cancer cells such as melanoma. The idea behind the insertion of two transgenes is that the targeted delivery of these potent co-stimulatory proteins may improve anti-cancer immune responses to the melanomas and diminish potential undesired off-target effects [32,34-36,43-45]. To do this, the virus AdV-D24-ICOSL-CD40L replicates in cancer cells whereby the cancer cells are killed via lysis and new virions are released along with the co-stimulatory molecules. In principle, this process can continue as long as there are tumor cells remaining. Previous preclinical data showed that the deletion of 24-bp (D24) of the vector results in a selective replication in cancer cells [37,46]. Specific replication properties of our vectors (AdV-D24-ICOSL-CD40L and AdV-D24) were achieved by the deletion of 24-bp in E1A Conserved Region 2 (CR2). Indeed, the vector was also modified in the fiber knob region, where chimeric oncolytic adenovirus (chimera $5 / 3$, a hybrid Ad5/3 fiber) was engineered and serotype adenovirus 5 fiber knob domain was replaced by serotype knob 3 domain in order to enhance the infectivity in cancer cells $[47,48]$.

Furthermore, the virus was rescued by transfecting PacI-linearized pAdV-D24-ICOSLCD40L cosmid DNA into A549 cells. Plaques were passaged in A549 cells and their identity was confirmed by the restriction digestion of Hirt DNA with HindIII, BamHI and NdeI (Figure S1). Then, a large-scale amplification/purification of viruses was performed. A total of $1.6 \times 10^{12} \mathrm{VP}$ and $2.9 \times 10^{12} \mathrm{VP}$ were obtained for two separate batches. The ratio between $\mathrm{VP} / \mathrm{IU}$ (ratio $\mathrm{VP} / \mathrm{mL} / \mathrm{IU} / \mathrm{mL}$ ) was calculated and ranged from 11 to 13 or 21 (mean ratio: 1:17) in different stocks. These experiments confirmed that a novel oncolytic adenovirus AdV-D24-ICOSL-CD40L expressing two co-stimulatory transgenes was successfully engineered, purified and produced in a larger scale for further preclinical studies.

\subsubsection{Whole Genome Sequencing of the Vector AdV-D24-ICOSL-CD40L}

The presence of incorporated genetic modification in the genome (D24, fiber $5 / 3$, ICOSL, CD40L) of newly engineered oncolytic vector AdV-D24-ICOSL-CD40L and the genetic stability were assessed by the sequencing of the entire genome of $\mathrm{CsCl}$-purified vector using NGS (Illumina). Whole genome sequencing of the vector AdV-D24-ICOSLCD40L revealed the presence of the 24-bp deletion in the E1A CR2 domain, the CMV-ICOSL- 
IRES-CD40L expression cassette inserted in place of the E3 region and the hybrid Ad5/3 fiber (Figure 1). Additionally, the analysis of sequence coverage did not detect the presence of any virus sub-population with a genome characterized by a major rearrangement (large insertion or deletion). The results confirmed the presence of inserted transgenes in the viral genome and the genetic stability.

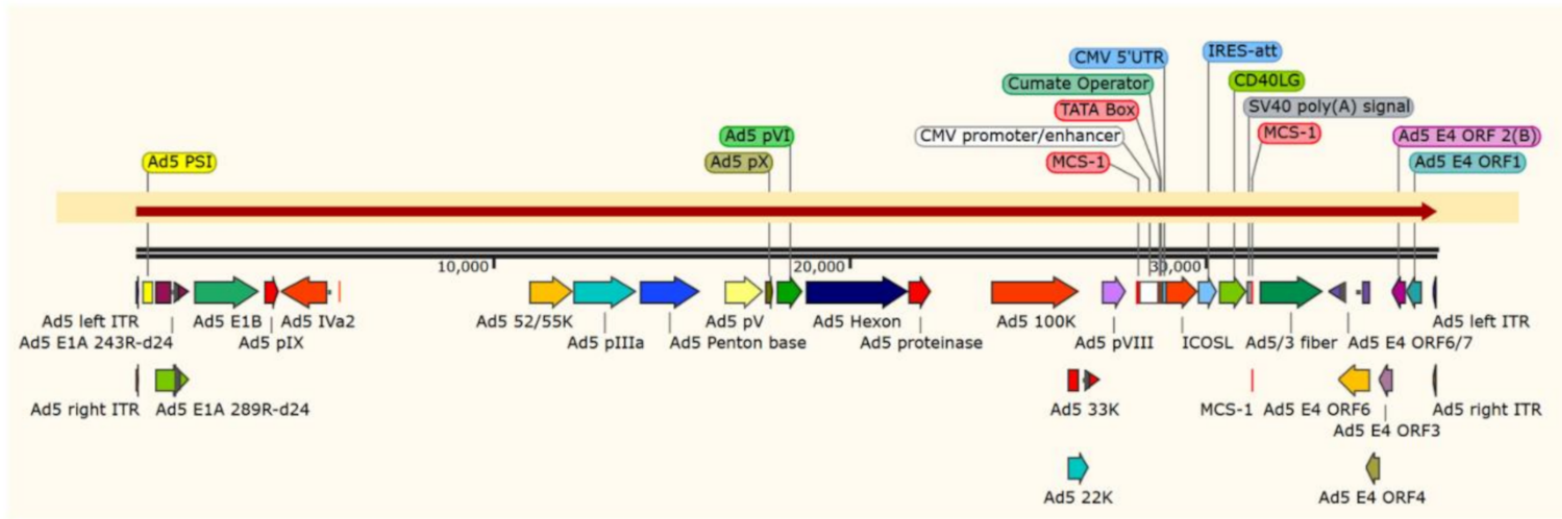

Figure 1. Viral DNA extraction from CsCl-purified virus particles using proteinase K. The AdV-D24-ICOSL-CD40L sequencing library was made using a Nextera XT kit (Illumina). The size of the library was assessed by high-resolution agarose gel electrophoresis. The presence of the 24-bp deletion in the E1A CR2 domain, the CMV-ICOSL-IRES-CD40L expression cassette inserted in place of the E3 region and the hybrid Ad5/3 fiber were confirmed. Analysis of sequence coverage did not detect the presence of a virus sub-population with a genome characterized by a major rearrangement such as a large insertion or deletion. The figure was generated by SnapGene ${ }^{\circledR}$.

\subsubsection{Expression of CD40L and ICOSL by the Vector AdV-D24-ICOSL-CD40L}

Since CD40L and ICOSL exert a role in activation of anti-tumor immune responses via the activation of $\mathrm{T}$ cells, we decided to incorporate these two transgenes into the viral backbone. The insertion of both co-stimulatory molecules under the exogenous promoter will allow the virus AdV-D24-ICOSL-CD40L to induce robust and long-lasting immune responses. Therefore, we confirmed, in both human melanoma cell lines MUG Mel-1 and MUG Mel-2, the expressions of CD40L and ICOSL following infection with AdV-D24ICOSL-CD40L by Western blotting (Figure 2C) and ELISA (Figure 3A,B) analysis after infection in A549 cells.

3.2. Expression of Coxsackie-Adenovirus Receptor (CAR) and Desmoglein-2 (DSG-2) Receptors in Human Melanoma Cell Lines MUG Mel-1 and MUG Mel-2

The therapeutic value of oncolytic viruses on melanoma was investigated using MUG Mel-1 and MUG Mel-2 cells by flow cytometry (Beckman-Coulter Cytomics FC500). The expression of adenovirus cell entry specific receptors, namely coxsackie-adenovirus receptor (CAR) and desmoglein-2 (DSG-2), were tested. The results show that the MUG Mel-1 cell line expressed a higher level of DSG-2 receptors (approximately 80\% of cells positive for the marker) compared to MUG Mel-2 (approximately 30\%). The expression of CAR receptors was much lower in both the cell lines (approx. 4\% for both MUG Mel-1 and MUG Mel-2) (Figure S2), thus, suggesting that both metastatic melanoma (Mug Mel-1) and melanoma from the skin (MUG Mel-2) can be targeted with oncolytic adenoviruses exhibiting affinity to DSG-2 receptors [49] for therapeutic treatment. 
A

MUG Mel-1
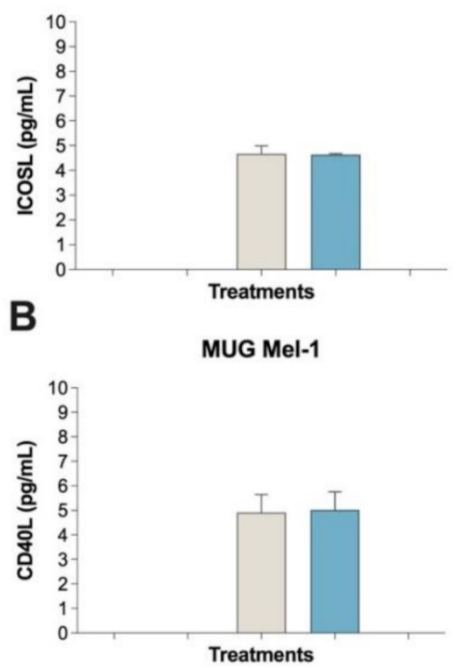

C

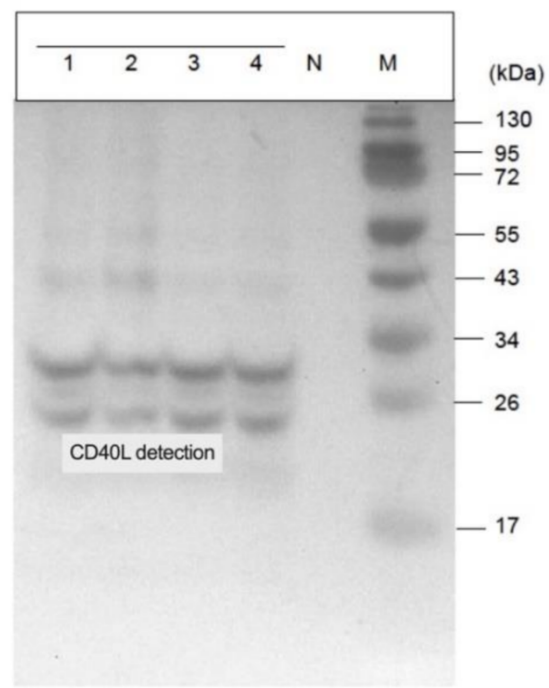

MUG Mel-2
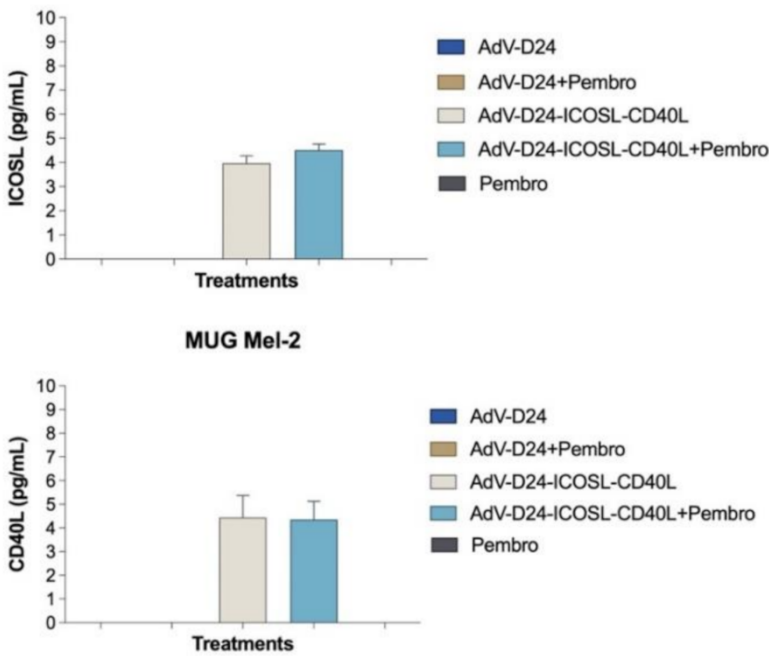

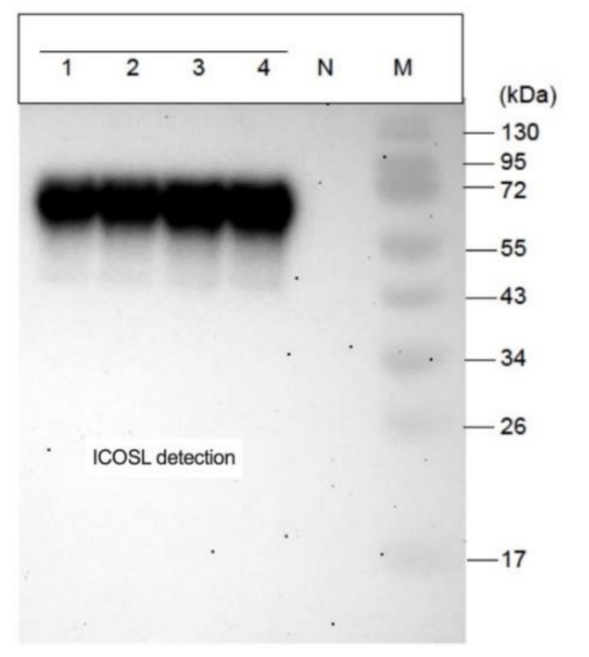

Figure 2. Evaluation of ICOSL and CD40L expression in tested melanoma cells. (A) ICOSL concentration was detected on supernatants collected $24-48 \mathrm{~h}$ after treatment using ELISA kit. (B) CD40L was detected from supernatants collected $24-48 \mathrm{~h}$ after treatment using an ELISA kit according to the manufacturer's instructions. Statistical analysis was carried out with a Mann-Whitney test to compare two groups (ns = not significant, $p>0.05$ ). (C) Transgene expression. The expression of ICOSL and CD40L from AdV-D24-ICOSL-CD40L was assessed by infecting A549 cells with the virus, harvesting proteins $48 \mathrm{~h}$ after the infection and detecting ICOSL and CD40L by Western blot. 1-4: protein extracts from A549 cells infected with AdV-D24-ICOSL-CD40L clones \#1-4. N: protein extract from non-infected A549 cells. M: PageRuler Prestained Protein Ladder (ThermoScientific \# 26616). ICOSL MW: $70 \mathrm{kDa}$ (due to heavy protein glycosylation); CD40L MW: $30 \mathrm{kDa}$. 

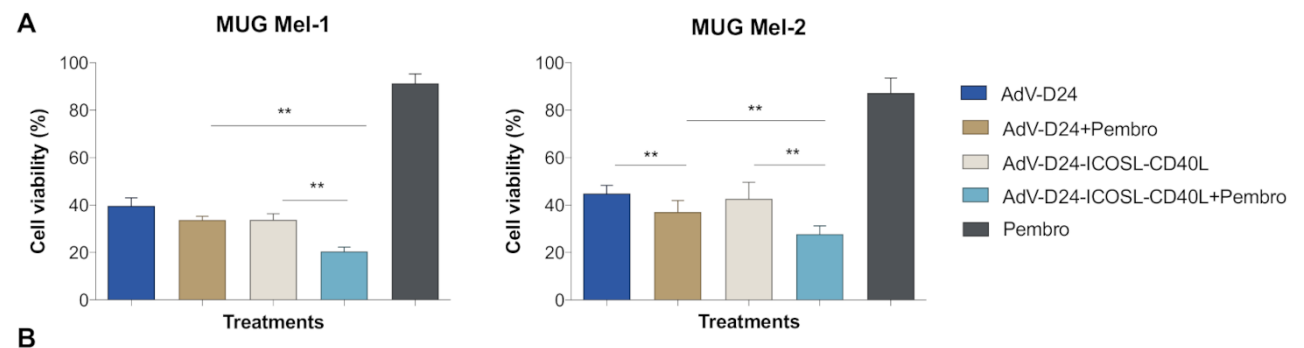

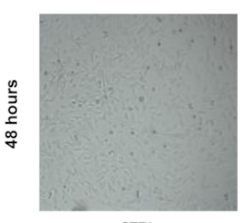

CTRL

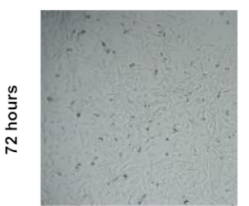

CTR

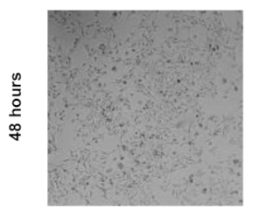

CTRL

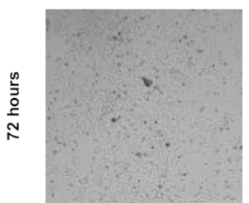

CTRL

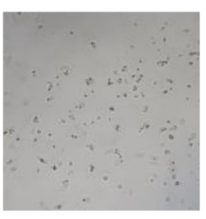

AdV-D24

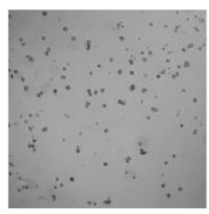

AdV-D24

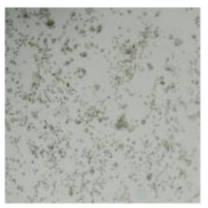

AdV-D24

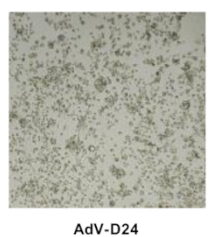

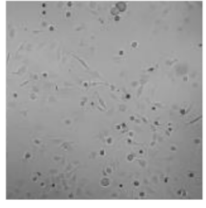

AdV-D24+ Pembro

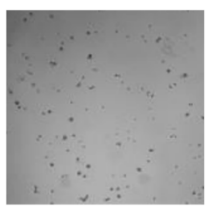

AdV-D24 + Pembro

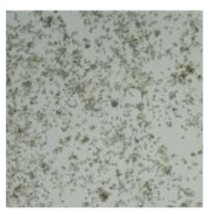

AdV-D24+ Pembro

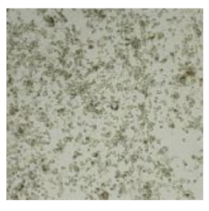

AdV-D24+ Pembro

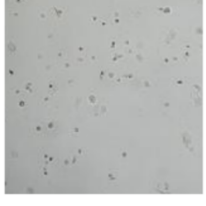

AdV5-D24-ICOSL-CD40L

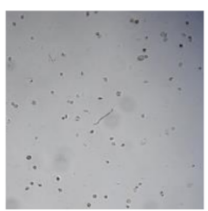

AdV-D24-ICOSL-CD40L



AdV-D24-ICOSL-CD $40 L$

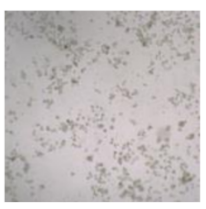

AdV-D24-ICOSL-CD40L

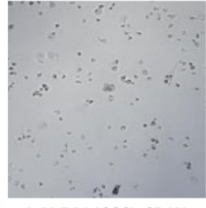

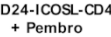

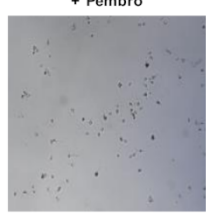
AdV-D24-ICOSL-CD40L

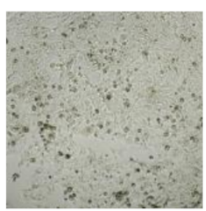

AdV-D24-ICOSL-CD 40

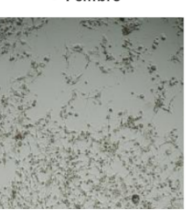

AdV-D24-ICOSL-CD40L



Pembro

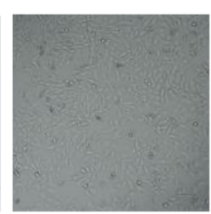

Pembro

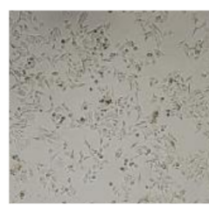

Pembro

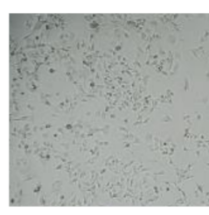

Pembro

Figure 3. Evaluation of cell viability by MTS assay (cell cytotoxicity assay). (A) Cell viability was evaluated $72 \mathrm{~h}$ post-infection with AdV-24-ICOSL-CD40L and AdV-24 at the concentration of $100 \mathrm{VP} /$ cell and combination with anti-PD1 (pembrolizumab). Data are expressed as the percentage of viable cells according to MTS cell viability assay protocol (CellTiter $96^{\circledR}$ AQueous One Solution Cell Proliferation Assay, Promega). Statistical analysis was carried out with a Mann-Whitney test to compare two groups $(p>0.05)$. (B,C) Microscopic photographs visualizing the morphology and cytopathic effect (CPE) of the cells representing the investigated groups and assessed by MTS assay at 48 and $72 \mathrm{~h}$ post-infection (magnification $\left.10 \times){ }^{* *}=p \leq 0.001\right)$.

\subsection{Evaluation of Cell Viability by MTS Assay (Cell Cytotoxicity Assay)}

The infectivity and the cell killing activity of AdV5/3-D24-ICOSL-CD40L was derived with an MTS cell viability assay on human and murine melanoma cell lines. As the control, the oncolytic adenovirus AdV-D24 which does not present neither the chimeric serotype nor the double transgene insertion was used. Following the infection of MUG Mel-1 and MUG Mel-2 cell lines with AdV-24-ICOSL-CD40L and AdV-D24 at the concentration of $100 \mathrm{VP} /$ cell, alone and/or in combination with anti PD-1 (pembrolizumab) or only in the presence of pembrolizumab, a reduction in cell viability (expressed in the percentage $(\%)$ of viable cells) was observed. The obtained data indicate that the combination therapy of the double transgene vector AdV-24-ICOSL-CD40L with anti PD-1 was the most effective treatment in both cell lines (cell viability was 20.5\% and 28\% for MUG Mel-1 and MUG Mel-2, respectively). In contrast, the monotherapy with the pembrolizumab was the least effective (91.3\% and 87\% for MUG Mel-1 and MUG Mel-2, respectively) compared to other 
tested regimes. Indeed, the combination of AdV-24 and anti PD-1 also showed enhanced cell killing activity over the virus itself: 33.7\% (MUG Mel-1) and 37.14\% (MUG Mel-2) (Figure 3A). These in vitro results demonstrated that both vectors administered together with pembrolizumab exhibit higher anti-cancer activity with respect to monotherapies. Nevertheless, the efficacy of the combination therapy was higher for the double transgene vector, suggesting higher anti-cancer potency due the presence of the co-stimulatory transgenes. Interestingly, the same trend has been also observed in B16V murine cells, where usually human oncolytic adenoviruses do not optimally replicate, hence the antitumor efficacy of AdV-D24 (42.7\%), pembrolizumab (94.7\%), and AdV-D24-ICOSL-CD40L (41\%) individually or in different combinations on B16V cell line was lower than in human melanoma cells (40.4\% and 37.3\% for AdV-24 plus anti PD-1 and AdV-D24-ICOSL-CD40L plus anti PD-1, respectively) (Figure S3). Notably, both human and murine cells treated only with pembrolizumab showed the least reduction in cell viability (Figure 3A) suggesting that the antibody alone does not have an impact on the cancer cell growth. In line with that, microscopic photographs at 48 and $72 \mathrm{~h}$ post-infection (magnification $10 \times$ ), show changes in the morphology of cells, confirming the anticancer and cytopathic effect (CPE) on cells treated with the different groups (infected with AdV-D24 or AdV-D24-ICOSLCD40L only or with pembrolizumab or only treated with pembrolizumab) (Figure 3B,C), respectively), thus suggesting that AdV5/3-D24-ICOSL-CD40L alone and or in combination with pembrolizumab could exert an antitumor effect against melanoma.

\subsection{Immunogenic Cell Death Assessment}

To evaluate whether the treatments can trigger immunogenic cell death, the appearance of specific markers, such as the exposure of calreticulin on cell surface and the extracellular release of ATP and HMGB1 [50-52], was measured on human melanoma cells treated with (i) AdV-D24 (100 VP/cell), (ii) AdV-D24-ICOSL-CD40L (100 VP/cell), (iii) anti

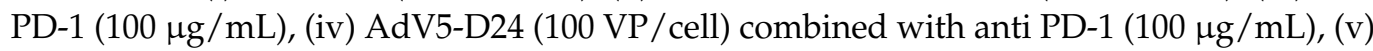
Ad5V-D24-ICOSL-CD40L (100 VP/cell) combined with anti PD-1 (100 $\mu \mathrm{g} / \mathrm{mL})$. The highest immunogenic cell death was observed in the cells infected with AdV-D24-ICOSL-CD40L and treated with pembrolizumab compared to other tested groups $(p<0.001, p<0.05$, $p<0.05$, respectively CRT, ATP and HMGB-1 for MUG Mel-1: AdV-D24-ICOSL-CD40L plus pembrolizumab vs. AdV-D24-ICOSL-CD40L; $p<0.0001, p<0.001$ for, respectively CRT, ATP for MUG Mel-2: AdV-D24-ICOSL-CD40L plus pembrolizumab vs. AdV-D24ICOSL-CD40L) (Figure 4), while the treatment of cells with only pembrolizumab seemed to not influence the immunogenic cell death program. Nevertheless, it should be also noted that immunogenic cell death markers were significantly higher in cells infected with AdV-D24 and treated with pembrolizumab compared to cells infected only with AdV-D24 in both the cell lines MUG Mel-1 and MUG Mel-2 $(p<0.001$ for CRT for MUG Mel-1: AdV-D24 plus pembrolizumab vs. AdV-D24; $p<0.05, p<0.05$ for, respectively, CRT and HMGB-1 for MUG Mel-2: AdV-D24 plus pembrolizumab vs. AdV-D24) (Figure 4). In line with these observations on the immunogenic cell death (ICD) studies, the results on B16V showed a similar trend for the studied markers: ATP and calreticulin (Figure S1).

Moreover, since this study is limited to the tumor cell response in vitro without the contribution of immune cells that play a crucial role in the development and maintenance of ICD, we concluded that the virus combined with pembrolizumab were able to block the growth of tumor cells and induce immunogenic cell death according to another mechanism different than the immune cell-mediated one. Finally, these findings highlight that the extent of immunogenic cell death was more remarkable $(p \leq 0.05)$ for the combination of the double transgene vector with anti PD-1, suggesting their immunomodulatory properties in vitro. 
A

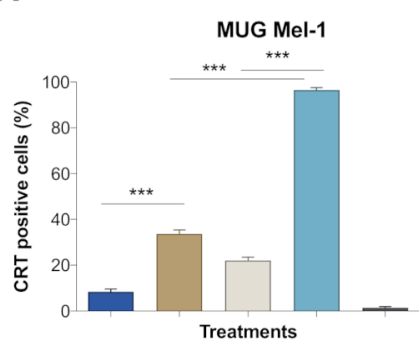

B



MUG Mel-1

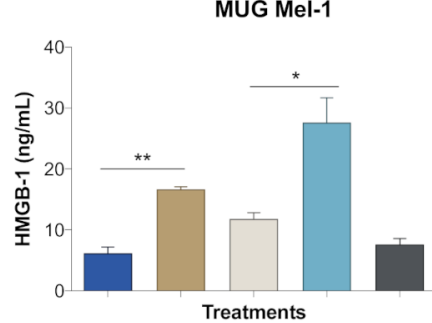

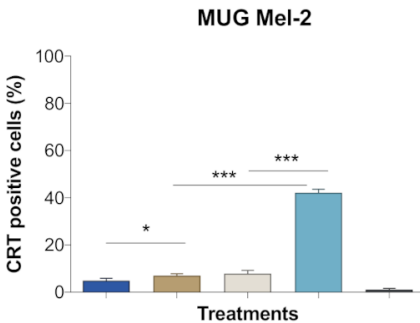

AdV-D24

AdV-D24+Pembro

AdV-D24-ICOSL-CD40L

AdV-D24-ICOSL-CD4OL+Pembro

- Pembro

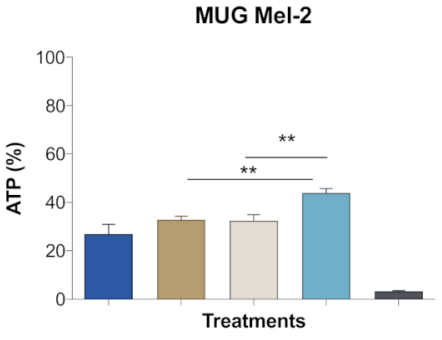

MUG Mel-2

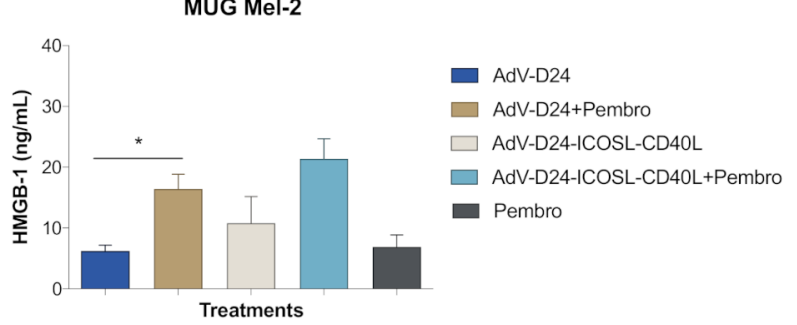

Figure 4. Immunogenic cell death assessment. (A) Evaluation of calreticulin (CRT) exposure by melanoma cell lines after treatment with oncolytic adenoviruses AdV-24-ICOSL-CD40L and AdV-D24, and in combination with anti PD-1. CRT exposure was measured $48 \mathrm{~h}$ post-treatments with anticalreticulin antibody staining AlexaFluor ${ }^{\circledR} 488$ and subsequent flow cytometry analysis (BeckmanCoulter Cytomics FC500). (B) Assessment of ATP release after the treatment. ATP concentration in a supernatant was evaluated $72 \mathrm{~h}$ after infection with CellTiter-Glo ${ }^{\circledR}$ Luminescent Cell Viability Assay ATP detection kit by Promega. (C) Evaluation of high-mobility group box 1 (HMGB-1) release after treatment with oncolytic adenoviruses and the combination with anti PD-1. HMGB-1 level was measured from the supernatant collected $72 \mathrm{~h}$ after infection with ELISA kit (MBL International, Woburn, MA, USA), according to manufacturer's dispositions. Statistical analysis was carried out with a Mann-Whitney test to compare two groups $\left({ }^{*}=p \leq 0.05 ;{ }^{* *}=p \leq 0.001,{ }^{* * *}=p \leq 0.0001\right)$.

3.5. Antitumor Efficacy of AdV-D24-ICOSL-CD40L and the Combination Therapy with Anti PD-1 Antibody in Murine Melanoma B16V Allograft Immunocompetent C57BL/6 Model

The ability of the combination treatment using oncolytic viruses with pembrolizumab to enhance anti-cancer potency and induce immunogenic cell death were investigated in vivo. To this aim, we used the C57BL/ 6 as the immunocompetent mouse model rather than immunodeficient xenograft alternative models [53] since we aimed to elucidate the involvement and contribution of the immune system to the efficacy of our combination therapy. Syngeneic tumor models (C57BL/6 or BALB/c mice) are the oldest and most commonly utilized preclinical models to evaluate anticancer therapeutics. The fully immunocompetent models are useful in the assessment of immuno-oncology agents (ICIs, oncolytic vectors) in order to study the development of antitumor immune responses, plus they do not require the adoptive transfer of immune cells [54,55].

Animal studies in syngeneic melanoma mouse model were performed in order to evaluate the possible antitumor effects triggered by the different treatments planned, according to the schedule presented in Table 1. 
Interestingly, the highest decrease in tumor volume growth rate was detected in the group of mice treated with AdV-D24-ICOSL-CD40L in combination with anti PD-1 $\left(31.3 \mathrm{~mm}^{3}\right)$ with a significant difference when compared to the group treated with AdVD24 plus anti PD-1 $\left(244 \mathrm{~mm}^{3}\right)(p<0.001$ for tumor volume) and the group treated with anti PD-1 $\left(445 \mathrm{~mm}^{3}\right.$ ) (Figure 5A,B). Nevertheless, also the mice treated only with the virus AdV-D24-ICOSL-CD40L resulted in significant tumor volume eradication $\left(85 \mathrm{~mm}^{3}\right.$, $p<0.05)$ compared to the group with anti-PD-1. Additionally, the vector AdV-D24 alone or in combination with anti PD-1 antibody also showed anticancer efficacy in comparison to anti-PD1 antibody alone, where no effect was detected thus suggesting that the B16V melanoma seems to be refractory to the ICI therapy $\left(261 ; 244 ; 445 \mathrm{~mm}^{3}\right.$, respectively for AdV-D24, AdV-D24 plus anti PD-1 and PD-1 alone). However, the most effective regimen in terms of tumor volume was the treatment with AdV-D24-ICOSL-CD40L in combination with anti PD-1 antibody on day 6 and day 21 (Figure 5C,D) thus indicating the most significant antitumor activity compared to other groups.

Notably, all the mice well tolerated the treatment and no signs of toxicity and weight loss were detected during the treatment course, thus justifying the benefits in survival profile, especially for the group of mice treated with viruses and anti-PD1 antibody. Remarkably, the group injected with AdV-D24-ICOSL-CD40L and treated in combination with anti PD-1 antibody resulted in 100\% survival (Figure 5E,F), thus suggesting it is the most efficacious therapy with a safe profile.

It has been shown that cancer cell death can be immunogenic or non-immunogenic. Immunogenic cell death (ICD) comprises changes in the structure of the cell surface and leads to the release of proimmunogenic factors. Subsequently, it attracts APCs to take up tumor antigens, process them and finally elicit an anti-tumor immune response (specific anti-tumor T cells). ICD can be evaluated by the presence of ICD biomarkers such as calreticulin (CRT) in the outer plasma membrane, followed by the extracellular release of high-mobility group box 1 protein (HMGB1) and adenosine triphosphate (ATP). Therefore, in our studies we have assessed the expression of the ICD markers in order to study the immunogenicity caused by the treatment. In fact, the in vitro model did not mimic optimal conditions due to the lack of immune system, which is crucial in the development of ICD [56]. Therefore, we conduced ex vivo analyses towards further assessment of the ICD based on the tumor tissue extracted from the treated mice. Our ex vivo results showed that the combinatory therapy (oncolytic adenovirus plus anti PD-1) resulted in the most profound expression of the ICD markers (ATP: AdV-D24-ICOSL-CD40L + pembro vs. pembro, $p<0.05$ ) (Figure $5 \mathrm{G}, \mathrm{H}$ ).

Our findings indicate that the combination therapy might boost anti-cancer immune responses and highlight the potential of the oncolytic vectors as an immunosensitizing agent for combination therapies with checkpoint inhibitors, as also reported by Ranki et al. [57]. Importantly, this effect can be further enhanced by arming oncolytic vectors with properly selected co-stimulatory molecules such as CD40L and ICOSL. 
A

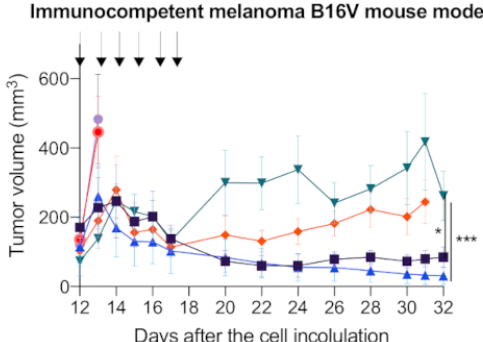

C

Immunocompetent melanoma B16V mouse model, Day 6

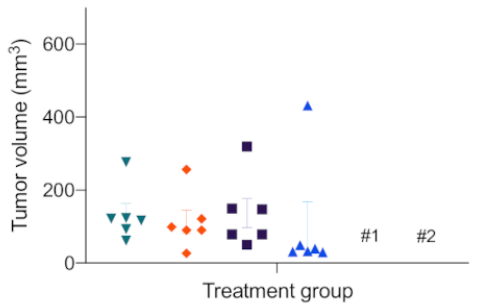

E



G

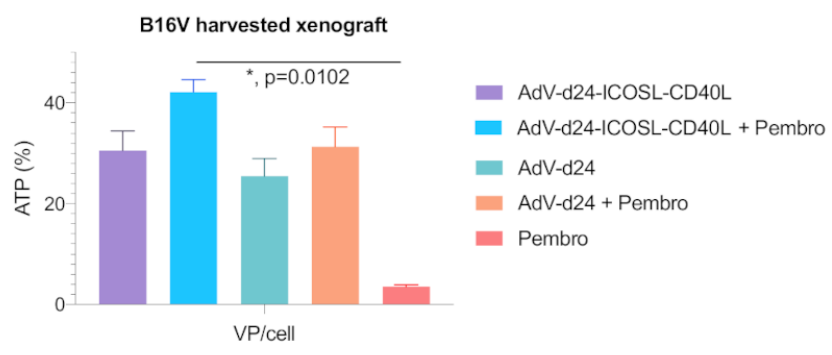

Control (\#2)

- anti-PD1 (\#1)

V AdV-d24

- AdV-d24-ICOSL-CD40L

- AdV-d24+anti-PD1

$\Delta \quad$ AdV-d24-ICOSL-CD40L+anti-PD
B Immunocompetent melanoma B16V mouse model

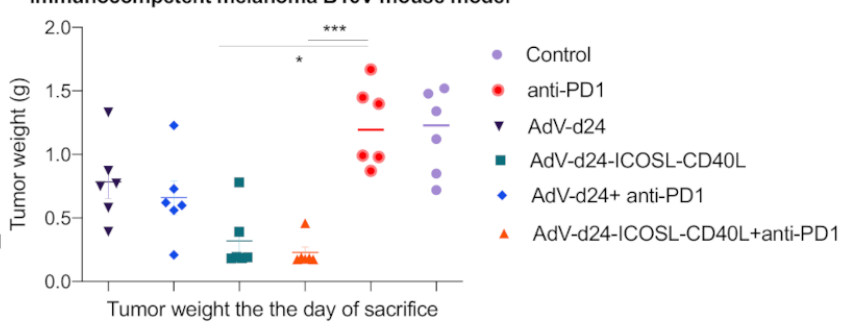

D

Immunocompetent melanoma B16V mouse model, Day 20

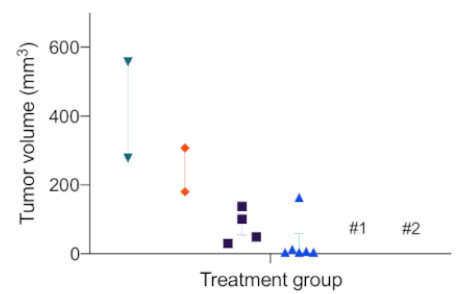

Control (\#2)

- anti-PD1 (\#1)

- AdV-d24

- AdV-d24-ICOSL-CD40L

- AdV-d24+anti-PD1

4 AdV-d24-ICOSL-CD40L+anti-PD1

Immunocompetent melanoma B16V mouse model

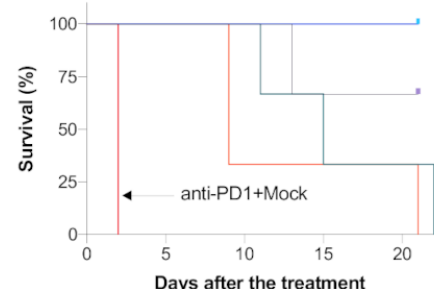

Contro

- anti-PD

- AdV-d24

- AdV-d24-ICOSL-CD40L

- AdV-d24+anti-PD1

- AdV-d24-ICOSL-CD40L+anti-PD1

H

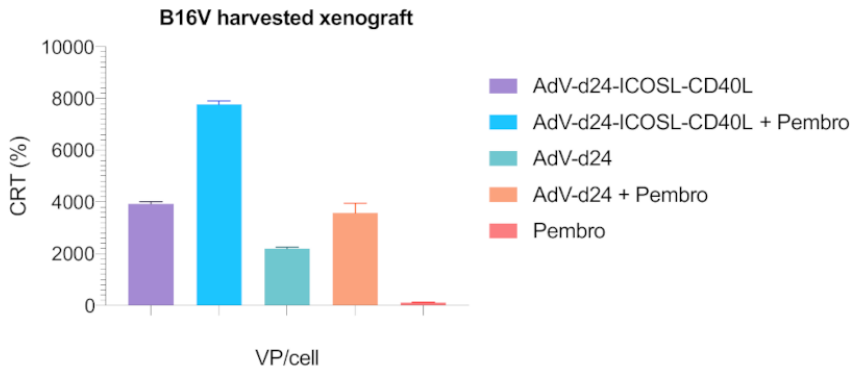

Figure 5. Antitumor efficacy of oncolytic vectors and the combination therapy with anti PD-1 antibody in murine melanoma B16V $\left(1 \times 10^{6}\right.$ cells / flank, one mouse had 2 tumors $/ 6$ tumors per group $)$ xenograft immunocompetent C57BL/ 6 model. (A) Tumor volume $\left(\mathrm{mm}^{3}\right)$ measured through the study. The treatment was performed once per day on days 1-6. The mice were treated according to the scheme (Table 1) with viruses (i.t.) and anti PD-1 antibody (i.v.) (B) At the end of the study, mice were sacrificed and tumors harvested for weight assessment. (C,D) Tumor volume measurement on days 6 and 20. (E) Body weight measurements throughout the study. (F) Survival profile was calculated by Kaplan-Meier test. (H) Evaluation of CRT exposure after the treatment with oncolytic adenoviruses AdV-24-ICOSL-CD40L and AdV-D24, and in combination with anti PD-1. CRT exposure was measured in the end of the study (after mice sacrifice) with anti-calreticulin antibody staining and subsequent flow cytometry analysis (Beckman-Coulter Cytomics FC500). (G) Assessment of ATP release after the treatment. ATP concentration from the tumors was evaluated in the end of the study (after mice sacrifice) with CellTiter-Glo ${ }^{\circledR}$ Luminescent Cell Viability Assay ATP detection kit by Promega. Error bars, mean \pm SEM, ${ }^{*}=p \leq 0.05$, $* * *=p \leq 0.0001$.

\section{Discussion}

The low 5-year survival rate in metastatic melanoma (around 20\%) [58] suggests that the use of immune checkpoint inhibitors (ICIs) such as pembrolizumab has provided benefits only in a small number of cases [4,59]. Indeed, for the majority of the patients, newer treatment options, either alone or in combination with already approved treatment 
modalities, are urgently required [60]. Although ICIs have enhanced the survival of some melanoma cancer patients by a few years, there are still many cases resistant to ICI treatments [61]. Furthermore, about $25 \%$ of the responders, despite ongoing therapy, develop relapses due to treatment resistance $[25,62]$. Nevertheless, metastatic melanoma responds better to immunotherapy compared to conventional chemotherapeutic drugs and radiotherapy [63-65]. Hence, combined treatment with novel oncolytic adenovirus and ICIs induces complementary antitumor response that could actually lead to more favorable therapeutic outcomes [66-69]. Indeed, pre-clinical and clinical findings show that oncolytic vectors can trigger anti-tumor immunity and increase immune cell infiltration (including cytotoxic CD8+ T cells) into the tumor microenvironment (TME). Priming with oncolytic vector can switch a "cold" TME into a "hot" one in terms of the infiltration of various innate and adaptive immune cell subsets into a tumor lesion. Therefore, this observation sets the stage for combination therapy with ICIs as they are most effective in a TME with a presence of lymphocytic infiltrates [7]. Indeed, it is conceivable that oncolytic vectors plus ICIs will be used as a combination therapy for multiple types of cancers, including melanoma, thus a considerable spectrum of various genes has already been integrated in oncolytic adenoviruses to enhance immune stimulation including antigen presentation, $\mathrm{T}$ cell priming or counterplay with immunosuppression. Such critical concepts have the potential to play a promising future role as enablers of immunotherapies involving oncolytic vectors [70,71].

Although extensive studies have been conducted to improve melanoma therapy [72,73], the treatment of advanced melanoma malignancies still presents a few challenges such as the need to refine the efficacy, safety and tolerability of ICIs. Oncolytic viruses (OVs) have demonstrated their ability to provide a synergistic anticancer effect in combination with ICIs, chemotherapy and radiotherapy $[15,50,71,74-76]$. Although OVs show clinical promise in already immunogenic malignancies and a safe profile, clinical response rates are inconsistent [77]. Therefore, the efficacy of oncolytic vectors needs to be strengthened in order to translate them as a mainstream in cancer therapy. One of the strategies to enhance the therapeutic efficacy of OVs is their use as primers for other immunotherapies. To this point, genes encoding for immunomodulatory proteins are the most commonly studied for arming oncolytic vectors and have the potential to widen the applications of immunooncology (IO) in cancers such as melanoma [69]. Therefore, we designed a strategy to engineer a newly oncolytic vector AdV-D24-ICOSL-CD40L and investigated its anti-tumor activity in combination with anti PD-1 in in vitro and in vivo immunocompetent mouse model engrafted with murine B16V melanoma cells. Moreover, the novel AdV-D24-ICOSLCD40L has been produced to purposefully infect cancer cells through the DSG-2 receptor. Indeed, in order to target tumor cells, the expression of the adenovirus receptor on the tumor cell surface is crucial [78,79]. Hence, it has been reported that different adenovirus serotypes bind various receptors on the cell of which the expression can vary between tumor types. Moreover, adenovirus 5 infects the cells through the CAR receptor, while adenovirus 3 binds to the DSG-2 and CD46 instead [79]. Accordingly, in our experiments, the expression level of the primary entry receptors has been assessed in both tested melanoma cell lines: MUG Mel-1 and MUG Mel-2. The obtained results demonstrated that the MUG Mel-1 cell line expressed a higher level of DSG-2 receptors (approx. 80\% of the cells express DSG-2) compared to MUG Mel-2 (approx. 30\%). In turn, the expression of the CAR receptor was low in both the cell lines (4.4\% and 4.0\%, respectively for MUG Mel-1 and MUG Mel-2), thus supporting the rationale for the treatment of melanoma using oncolytic vector AdV-D24-ICOSL-CD40L [49].

Moreover, cell viability assays, carried out with both human melanoma cell lines, revealed that combination therapy using AdV-D24-ICOSL-CD40L with pembrolizumab led to higher anti-cancer activity compared to the other tested groups. Interestingly, cell viability was affected the least in all of the in vitro models treated only with anti PD-1 antibody. This is in agreement with our previous studies showing that the addition of 
pembrolizumab along with oncolytic adenovirus AdV5/3-D24-GM-CSF in SK-Mel-28 cells led to a stronger anti-tumor effect in comparison to single therapies [80].

It is well known that oncolytic adenoviruses are able to induce immunogenic cancer cell death (ICD) and the release of tumor specific antigens for APC cells, triggering the priming of potent tumor-specific immunity [70]. Hence, the combination of oncolytic vectors with other therapeutic agents has the potential for enhanced anticancer efficacy and the development of antineoplastic immunity, thus representing a powerful tool to overcome the major obstacle of an immune suppressive microenvironment and the subsequent induction of anti-cancer immune responses [81]. While assessing the extent of ICD, it has been found that the level of calreticulin exposure (CRT positive cells in \%), and ATP (in \%) and HMGB-1 (in \%) release were significantly elevated in both cell lines treated with AdV-D24-ICOSL-CD40L in combination with pembrolizumab as compared to the other groups, except for the HMGB-1 level in MUG Mel-2 cells where the level was higher, but the difference was not significant $(p<0.001, p<0.05, p<0.05$ for, respectively CRT, ATP and HMGB-1 for MUG Mel-1: AdV-D24-ICOSL-CD40L plus pembrolizumab vs. AdV-D24-ICOSL-CD40L; $p<0.0001, p<0.001$ for, respectively CRT, ATP for MUG Mel-2: AdV-D24-ICOSL-CD40L plus pembrolizumab vs. AdV-D24-ICOSL-CD40L) and this trend was also confirmed in B16V cells (ATP: AdV-D24-ICOSL-CD40L + pembro vs. pembro, $p<0.05)$. Interestingly, in line with our findings, it is reported by the literature that the combination therapy of oncolytic adenoviruses with chemotherapeutic drugs and ICIs resulted in the enhanced expression of calreticulin on the cancer cell surface and a higher release of both ATP and HMGB-1 markers in mesothelioma and melanoma therapies $[50,80]$. Indeed, it is postulated that ICD constitutes a prominent pathway for the activation of the immune responses against cancer, which in turn affect the long-term success of anti-cancer therapies along with the long-lasting protective anti-tumor immunity [51]. Taken together, these results may suggest an enhanced cytotoxic immune effect induced by AdV-D24ICOSL-CD40L and pembrolizumab compared to other groups. Certainly, the impact of these effects has to be further investigated in additional mouse models (e.g., humanized) by focusing on immune responses, the infiltration of immune cells to the injected lesions and $\mathrm{T}$ cell phenotyping, but this was not the primary aim of this work.

The interplay among cancer cells, immune cells and anticancer drugs is complex. Indeed, the success of immunotherapy has changed the practice of cancer treatment tremendously [82]. However, there are still many challenges, such as drug resistance, the assessment of combinatory therapies, biomarker discovery, the prediction of adverse events in preclinical settings and others. To overcome these challenges, it is crucial to develop reliable preclinical mouse models that recapitulate the clinical features, biological heterogeneity, and immune microenvironment of human cancers [83]. Selecting the right mouse model to study these interactions is a key step for the development of immunotherapies [84]. Therefore, in order to assess the anti-cancer potential and clinical benefits of the combination therapy of oncolytic adenoviruses and ICIs, we have developed an immunocompetent melanoma B16V mouse model.

Evaluation of antitumor efficacy of AdV-D24-ICOSL-CD40L, AdV-D24 and the combination therapy with anti PD-1 antibody in murine melanoma B16V allograft immunocompetent C57BL / 6 model revealed maximum and significant reduction in tumor volume in mice treated with AdV-D24-ICOSL-CD40L combined with anti PD-1 antibody. Remarkably, the combination therapy resulted in $100 \%$ survival and no loss in body weight. This is a promising result, showing that the investigated combination treatment regimen might extend melanoma cancer patient survival and present a well-tolerated safety profile. In addition, the ex vivo investigation of ICD profile evaluated on explanted murine tumor tissues confirmed that the combinatory therapy resulted in the most profound expression of the ICD markers. Nevertheless, despite these findings, our in vivo study has some limitations as the human oncolytic adenoviruses are not optimally replicating in murine tissue $[85,86]$. The efficient replication of human adenoviruses in murine cells is significantly lower than in human cells, although infection can be detectable. However, the mechanisms 
for a low level of infectious virions remain unclear [87]. Therefore, this limitation could have affected the anti-cancer efficacy of the viruses itself in the B16V mouse model. Nevertheless, on the other hand, oncolytic viruses in addition to oncolytic properties exhibit the ability to induce anti-cancer immune responses, which can lead to the eradication of cancer cells. However, the strength of the selected model and the results is the presence of a fully functional immune system in C57BL/6 mice allowing for a proper induction, development and modulation of immune responses against cancer cells and the prevention of the inhibition of effector $\mathrm{T}$ cells.

In a similar study, a rational combination approach aimed at exploring the role of immunogenic oncolytic adenovirus Ad5/3-D24-GM-CSF with pembrolizumab in humanized A2058 melanoma huNOG mouse model showed a significant reduction in tumor volume compared to pembrolizumab treatment alone without lowering body weight [80]. Notably, another study by Bramante S. et al. evaluated the effects of $5 / 3$ chimeric oncolytic adenovirus coding for GM-CSF with low dose cyclophosphamide in in vitro and in vivo melanoma models [88] revealing a promising outcome following treatment with the oncolytic virus, thus advocating the need for more studies exploring the use of oncolytic viral therapy able to support the future design of clinical trials and to enhance the efficacy of cancer treatment, including melanoma. For instance, ongoing clinical trials are currently exploring the role of oncolytic virus ONCOS-102 in combination with ICIs in melanoma cancer cases refractory to the treatment (NCT03003676). In this phase 1 trial, the combination of ONCOS-102 and pembrolizumab has been tested in patients with advanced, unresectable melanoma who have had disease progress despite treatment with anti-PD1. It has been reported that 7 out of 20 patients treated with ONCOS-102 and pembrolizumab combination resulted in an ORR of 35\% by RECIST 1.1 criteria. Importantly, it was also observed that multiple non-injected lesions completely disappeared, indicating that ONCOS-102 can induce systemic anti-tumor immunity [89]. Interestingly, Frohlich et al. also reported that the oncolytic vector T-VEC showed the potential for synergistic anti-cancer effects to overcome the resistance of mucosal melanoma to ICI anti PD-1 [90]. Other studies also support combining ICIs with oncolytic vectors. Particularly, the combination of ICIs with T-VEC may result in a synergistic anti-cancer efficacy for patients with unresectable melanoma as a higher ORR and complete response rate (CRB) was reported compared to published studies on similar therapeutic regimens [28]. In another clinical study on metastatic melanoma patients, treatment with T-VEC showed a better durable response rate (continuous complete response or partial response lasting $\geq 6$ months) over subcutaneous GM-CSF (16.3\% vs. $2.1 \%$; $p<0.001)$. Importantly, responses were observed in both injected and untreated lesions. Indeed, when T-VEC was combined with ICIs, significantly improved response rates were reported (compared to monotherapies). Similar findings were observed with combinations of ICIs and other oncolytic vectors such as CAVATAK with ipilimumab [91].

These findings highlight the potential of the oncolytic vectors as an immunosensitizing agent for combination therapies with checkpoint inhibitors in a group of ICI refractory patients [57].

Nevertheless, the promising results obtained from the published studies on the efficacy and safety of oncolytic viruses for the treatment of different types of cancer (ovarian, mesothelioma, melanoma, etc.) underlines the need for further studies aimed at exploring the roles of different oncolytic viruses in both preclinical and clinical settings. To the best of our knowledge, ours is the first study investigating the oncolytic role of AdV-D24ICOSL-CD40L in melanoma preclinical studies. Even if this represents an open line of investigation, as further studies should be carried out to better elucidate the role of AdVD24-ICOSL-CD40L in melanoma, it represents a milestone for development. Future studies will leverage the oncolytic and immunomodulatory properties of AdV-D24-ICOSL-CD40L in combination with other anticancer drugs complementing the anti-cancer properties, such as adoptive $\mathrm{T}$ cell therapy (ACT) and bispecific antibodies (BsAb), in order to maximize treatment against cancer. 


\section{Conclusions}

We have successfully engineered a novel oncolytic adenovirus AdV-D24-ICOSLCD40L expressing co-stimulatory molecules: ICOSL and CD40L. Our preclinical results strengthen the notion that combination therapy of oncolytic adenovirus with ICIs may enhance anti-cancer efficacy and survival via targeted cancer cell lysis and the induction of immunogenic cell death in melanoma models. These data may provide important insights to further study the combination therapies in solid tumors. Future studies will assess the role of ICOSL and CD40L in tumor cells and support possible recommendations for the management and treatment of melanoma-bearing patients. Notably, we have proposed a new treatment strategy against melanoma to be further investigated.

Supplementary Materials: The following are available online at https:/ /www.mdpi.com/article/ 10.3390/pharmaceutics13040547/s1, Figure S1. Virus rescue and characterization. Viral DNA was extracted from AdV-D24-ICOSL-CD40L infected A549 cells according to the Hirt method. The identity of the virus was assessed by restriction digestion with HindIII A, BamHI and NdeI B. All restriction patterns of AdV-D24-ICOSL-CD40L \#1-2-3-4 vDNA match that of the PacI-digested cosmid pAdV-D24-ICOSL-CD40L, indicating the stability of the vector. The presence of the ICOSL-CD40L cassette in the vector was confirmed by the presence of the restriction fragments highlighted in green in the pictures. A: pAdV-D24-ICOSL-CD40L/PacI, B: pAdV-D24-ICOSL-CD40L, C: pAdVD24-ICOSL-CD40L/PacI, D: pAdV-D24-ICOSL-CD40L. The identity of the vector was confirmed by restriction digestion of Hirt DNA with HindIII, BamHI and NdeI; Figure S2. Expression of coxsackieadenovirus receptor (CAR) and desmoglein-2 (DSG-2) receptors in human melanoma cell lines MUG Mel-1 and MUG Mel-2, measured with flow cytometry with Beckman-Coulter Cytomics FC500. Data are expressed as percentage of cell positive for the marker; Figure S3. Evaluation of cell viability by MTS assay (cell cytotoxicity assay) and immunogenic cell death. A Cell viability was evaluated $72 \mathrm{~h}$ post-infection with AdV-D24-ICOSL-CD40L and AdV-D24 at the concentration of $100 \mathrm{VP} /$ cell and combination with anti-PD1 in murine B16V melanoma cell line. Data are expressed as percentage of viable cells according to MTS cell viability assay protocol (CellTiter $96^{\circledR}$ AQueous One Solution Cell Proliferation Assay, Promega). Immunogenic cell death assessment. B Assessment of ATP release after the treatment. ATP concentration in a supernatant was evaluated $72 \mathrm{~h}$ after infection with CellTiter-Glo ${ }^{\circledR}$ Luminescent Cell Viability Assay ATP detection kit by Promega. C Evaluation of CRT exposure by melanoma cell lines after treatment with oncolytic adenoviruses AdV-24-ICOSL-CD40L and AdV-D24, and in combination with anti PD-1. CRT exposure was measured $48 \mathrm{~h}$ post-treatments with anti-calreticulin staining and subsequent flow cytometry analysis (Beckman-Coulter Cytomics FC500). Statistical analysis was carried out with a Mann-Whitney test to compare two groups $\left(^{*}=p \leq 0.05 ;{ }^{* *}=p \leq 0.001\right)$.

Author Contributions: Conceptualization, M.G. and L.K.; methodology, M.G. and L.K.; software, M.G., L.K. and L.B.; validation, M.G., L.K. and L.B.; formal analysis, M.G., L.K. and L.B.; investigation, M.G. and L.B.; resources, M.G., L.B., M.S., M.W., S.S. (Stefano Salmaso), S.S. (Silke Schrom), B.R., K.W.P. and L.K.; data curation, M.G. and L.B.; writing-original draft preparation, L.K. and M.G.; writing, M.G., L.B., M.S., M.W., S.S. (Stefano Salmaso), S.S. (Silke Schrom), B.R., K.W.P. and L.K.; visualization, M.G., L.K. and L.B.; supervision, M.G. and L.K.; project administration, L.K., funding acquisition, L.K. All authors have read and agreed to the published version of the manuscript.

Funding: This publication is based upon work from COST Action CA 17140 "Cancer Nanomedicine from the Bench to the Bedside" supported by COST (European Cooperation in Science and Technology) (L.K., M.G.). L.K. was supported by the National Science Centre, Poland SONATINA (2019/32/C /NZ7/00156) and the National Institute of Public Health-National Institute of Hygiene, Poland (1BWBW/19). M.G. acknowledges PRID-J (Grant Number: GARO_SID19_02) funded by University of Padua. M.S. was supported by the Centre for Advanced Materials and Technologies, WUT, Poland and grant POB Biotechmed-1 no. 504/04496/1020/45.010406 (Excellence Initiative Research University).

Institutional Review Board Statement: Not applicable.

Informed Consent Statement: Not applicable.

Data Availability Statement: Not applicable. 
Conflicts of Interest: L.K. is an employee/shareholder in Targovax. The funders had no role in the design of the study; in the collection, analyses, or interpretation of data; in the writing of the manuscript, or in the decision to publish the results. The authors declare no other conflict of interest.

\section{References}

1. Yde, S.S.; Sjoegren, P.; Heje, M.; Stolle, L.B. Mucosal Melanoma: A Literature Review. Curr. Oncol. Rep. 2018, 20, 28. [CrossRef] [PubMed]

2. Rastrelli, M.; Tropea, S.; Rossi, C.R.; Alaibac, M. Melanoma: Epidemiology, risk factors, pathogenesis, diagnosis and classification. In Vivo 2014, 28, 1005-1011. [PubMed]

3. Elder, D.E.; Bastian, B.C.; Cree, I.A.; Massi, D.; Scolyer, R.A. The 2018 World Health Organization Classification of Cutaneous, Mucosal, and Uveal Melanoma: Detailed Analysis of 9 Distinct Subtypes Defined by Their Evolutionary Pathway. Arch. Pathol. Lab. Med. 2020, 144, 500-522. [CrossRef] [PubMed]

4. Bray, F.; Ferlay, J.; Soerjomataram, I.; Siegel, R.L.; Torre, L.A.; Jemal, A. Global cancer statistics 2018: GLOBOCAN estimates of incidence and mortality worldwide for 36 cancers in 185 countries. CA Cancer J. Clin. 2018, 68, 394-424. [CrossRef]

5. World Health Organization. World Cancer Report (PDF); WHO: Geneva, Switzerland, 2014.

6. Matthews, N.H.; Li, W.Q.; Qureshi, A.A.; Weinstock, M.A.; Cho, E. Epidemiology of Melanoma. In Cutaneous Melanoma: Etiology and Therapy; Ward, W.H., Farma, J.M., Eds.; Codon Publications: Brisbane, Australia, 2017. [CrossRef]

7. LaRocca, C.J.; Warner, S.G. Oncolytic viruses and checkpoint inhibitors: Combination therapy in clinical trials. Clin. Transl. Med. 2018, 7, 35. [CrossRef]

8. Munhoz, R.R.; Postow, M.A. Clinical Development of PD-1 in Advanced Melanoma. Cancer J. 2018, 24, 7-14. [CrossRef]

9. Imbert, C.; Montfort, A.; Fraisse, M.; Marcheteau, E.; Gilhodes, J.; Martin, E.; Bertrand, F.; Marcellin, M.; Burlet-Schiltz, O.; Peredo, A.G.; et al. Resistance of melanoma to immune checkpoint inhibitors is overcome by targeting the sphingosine kinase-1. Nat. Commun. 2020, 11, 437. [CrossRef]

10. Larkin, J.; Chiarion-Sileni, V.; Gonzalez, R.; Grob, J.J.; Cowey, C.L.; Lao, C.D.; Schadendorf, D.; Dummer, R.; Smylie, M.; Rutkowski, P.; et al. Combined Nivolumab and Ipilimumab or Monotherapy in Untreated Melanoma. N. Engl. J. Med. 2015, 373, 23-34. [CrossRef]

11. Robert, C.; Schachter, J.; Long, G.V.; Arance, A.; Grob, J.J.; Mortier, L.; Daud, A.; Carlino, M.S.; McNeil, C.; Lotem, M.; et al. Pembrolizumab versus Ipilimumab in Advanced Melanoma. N. Engl. J. Med. 2015, 372, 2521-2532. [CrossRef]

12. Chiu, M.; Armstrong, E.J.L.; Jennings, V.; Foo, S.; Crespo-Rodriguez, E.; Bozhanova, G.; Patin, E.C.; McLaughlin, M.; Mansfield, D.; Baker, G.; et al. Combination therapy with oncolytic viruses and immune checkpoint inhibitors. Exp. Opin. Biol. Ther. 2020, 20, 635-652. [CrossRef]

13. O'Bryan, S.M.; Mathis, J.M. Oncolytic Virotherapy for Breast Cancer Treatment. Curr. Gene Ther. 2018, 18, 192-205. [CrossRef]

14. Russell, S.J.; Peng, K.W.; Bell, J.C. Oncolytic virotherapy. Nat. Biotechnol. 2012, 30, 658-670. [CrossRef]

15. Kuryk, L.; Moller, A.W.; Jaderberg, M. Abscopal effect when combining oncolytic adenovirus and checkpoint inhibitor in a humanized NOG mouse model of melanoma. J. Med. Virol. 2019, 91, 1702-1706. [CrossRef]

16. Lin, C.Z.; Xiang, G.L.; Zhu, X.H.; Xiu, L.L.; Sun, J.X.; Zhang, X.Y. Advances in the mechanisms of action of cancer-targeting oncolytic viruses. Oncol. Lett. 2018, 15, 4053-4060. [CrossRef]

17. Liu, T.C.; Kirn, D. Gene therapy progress and prospects cancer: Oncolytic viruses. Gene Ther. 2008, 15, 877-884. [CrossRef]

18. Maroun, J.; Munoz-Alia, M.; Ammayappan, A.; Schulze, A.; Peng, K.W.; Russell, S. Designing and building oncolytic viruses. Future Virol. 2017, 12, 193-213. [CrossRef]

19. Lichty, B.D.; Breitbach, C.J.; Stojdl, D.F.; Bell, J.C. Going viral with cancer immunotherapy. Nat. Rev. Cancer 2014, $14,559-567$. [CrossRef]

20. Liu, Z.; Ravindranathan, R.; Kalinski, P.; Guo, Z.S.; Bartlett, D.L. Rational combination of oncolytic vaccinia virus and PD-L1 blockade works synergistically to enhance therapeutic efficacy. Nat. Commun. 2017, 8, 14754. [CrossRef]

21. Wang, X.; Teng, F.; Kong, L.; Yu, J. PD-L1 expression in human cancers and its association with clinical outcomes. Oncotargets Ther. 2016, 9, 5023-5039. [CrossRef]

22. Bommareddy, P.K.; Patel, A.; Hossain, S.; Kaufman, H.L. Talimogene Laherparepvec (T-VEC) and Other Oncolytic Viruses for the Treatment of Melanoma. Am. J. Clin. Derm. 2017, 18,1-15. [CrossRef]

23. Alsaab, H.O.; Sau, S.; Alzhrani, R.; Tatiparti, K.; Bhise, K.; Kashaw, S.K.; Iyer, A.K. PD-1 and PD-L1 Checkpoint Signaling Inhibition for Cancer Immunotherapy: Mechanism, Combinations, and Clinical Outcome. Front. Pharm. 2017, 8, 561. [CrossRef] [PubMed]

24. Rothermel, L.D.; Zager, J.S. Engineered oncolytic viruses to treat melanoma: Where are we now and what comes next? Expert Opin. Biol. Ther. 2018, 18, 1199-1207. [CrossRef] [PubMed]

25. Ribas, A.; Dummer, R.; Puzanov, I.; VanderWalde, A.; Andtbacka, R.H.I.; Michielin, O.; Olszanski, A.J.; Malvehy, J.; Cebon, J.; Fernandez, E.; et al. Oncolytic Virotherapy Promotes Intratumoral T Cell Infiltration and Improves Anti-PD-1 Immunotherapy. Cell 2017, 170, 1109-1119.e10. [CrossRef] [PubMed]

26. Zheng, M.; Huang, J.; Tong, A.; Yang, H. Oncolytic Viruses for Cancer Therapy: Barriers and Recent Advances. Mol. Ther. Oncolytics 2019, 15, 234-247. [CrossRef] [PubMed] 
27. Hwang, J.K.; Hong, J.; Yun, C.O. Oncolytic Viruses and Immune Checkpoint Inhibitors: Preclinical Developments to Clinical Trials. Int. J. Mol. Sci. 2020, 21, 8627. [CrossRef] [PubMed]

28. Sun, L.; Funchain, P.; Song, J.M.; Rayman, P.; Tannenbaum, C.; Ko, J.; McNamara, M.; Marcela Diaz-Montero, C.; Gastman, B. Talimogene Laherparepvec combined with anti-PD-1 based immunotherapy for unresectable stage III-IV melanoma: A case series. J. Immunother. Cancer 2018, 6, 36. [CrossRef]

29. Wang, B.; Jiang, H.; Zhou, T.; Ma, N.; Liu, W.; Wang, Y.; Zuo, L. Expression of ICOSL is associated with decreased survival in invasive breast cancer. PeerJ 2019, 7, e6903. [CrossRef]

30. Aspord, C.; Leccia, M.T.; Charles, J.; Plumas, J. Plasmacytoid dendritic cells support melanoma progression by promoting Th2 and regulatory immunity through OX40L and ICOSL. Cancer Immunol. Res. 2013, 1, 402-415. [CrossRef]

31. Flies, D.B.; Chen, L. The new B7s: Playing a pivotal role in tumor immunity. J. Immunother. 2007, 30, 251-260. [CrossRef]

32. Kuryk, L.; Moller, A.-S.W.; Jaderberg, M. Quantification and functional evaluation of CD40L production from the adenovirus vector ONCOS-401. Cancer Gene Ther. 2018, 26, 26-31. [CrossRef]

33. Liu, X.; Bai, X.F.; Wen, J.; Gao, J.X.; Liu, J.; Lu, P.; Wang, Y.; Zheng, P.; Liu, Y. B7H costimulates clonal expansion of, and cognate destruction of tumor cells by, CD8(+) T lymphocytes in vivo. J. Exp. Med. 2001, 194, 1339-1348. [CrossRef]

34. Zamarin, D.; Holmgaard, R.B.; Ricca, J.; Plitt, T.; Palese, P.; Sharma, P.; Merghoub, T.; Wolchok, J.D.; Allison, J.P. Intratumoral modulation of the inducible co-stimulator ICOS by recombinant oncolytic virus promotes systemic anti-tumour immunity. Nat. Commun. 2017, 8, 14340. [CrossRef]

35. Ara, A.; Ahmed, K.A.; Xiang, J. Multiple effects of CD40-CD40L axis in immunity against infection and cancer. Immunotargets Ther. 2018, 7, 55-61. [CrossRef]

36. Diaconu, I.; Cerullo, V.; Hirvinen, M.L.; Escutenaire, S.; Ugolini, M.; Pesonen, S.K.; Bramante, S.; Parviainen, S.; Kanerva, A.; Loskog, A.S.; et al. Immune response is an important aspect of the antitumor effect produced by a CD40L-encoding oncolytic adenovirus. Cancer Res. 2012, 72, 2327-2338. [CrossRef]

37. Koski, A.; Kangasniemi, L.; Escutenaire, S.; Pesonen, S.; Cerullo, V.; Diaconu, I.; Nokisalmi, P.; Raki, M.; Rajecki, M.; Guse, K.; et al. Treatment of Cancer Patients With a Serotype 5/3 Chimeric Oncolytic Adenovirus Expressing GMCSF. Mol. Ther. 2010, 18, 1874-1884. [CrossRef]

38. Sweeney, J.A.; Hennessey, J.P., Jr. Evaluation of accuracy and precision of adenovirus absorptivity at $260 \mathrm{~nm}$ under conditions of complete DNA disruption. Virology 2002, 295, 284-288. [CrossRef]

39. Guo, H.; Jiang, D.; Zhou, T.; Cuconati, A.; Block, T.M.; Guo, J.T. Characterization of the intracellular deproteinized relaxed circular DNA of hepatitis B virus: An intermediate of covalently closed circular DNA formation. J. Virol. 2007, 81, 12472-12484. [CrossRef]

40. Arad, U. Modified Hirt procedure for rapid purification of extrachromosomal DNA from mammalian cells. Biotechniques 1998, 24, 760-762. [CrossRef]

41. Marelli, G.; Howells, A.; Lemoine, N.R.; Wang, Y. Oncolytic Viral Therapy and the Immune System: A Double-Edged Sword against Cancer. Front. Immunol. 2018, 9, 866. [CrossRef]

42. Russell, L.; Peng, K.W.; Russell, S.J.; Diaz, R.M. Oncolytic Viruses: Priming Time for Cancer Immunotherapy. BioDrugs 2019, 33, 485-501. [CrossRef]

43. Pesonen, S.; Diaconu, I.; Kangasniemi, L.; Ranki, T.; Kanerva, A.; Pesonen, S.K.; Gerdemann, U.; Leen, A.M.; Kairemo, K.; Oksanen, M.; et al. Oncolytic immunotherapy of advanced solid tumors with a CD40L-expressing replicating adenovirus: Assessment of safety and immunologic responses in patients. Cancer Res. 2012, 72, 1621-1631. [CrossRef] [PubMed]

44. Fu, T.; He, Q.; Sharma, P. The ICOS/ICOSL Pathway Is Required for Optimal Antitumor Responses Mediated by Anti-CTLA-4 Therapy. Cancer Res. 2011, 71, 5445-5454. [CrossRef] [PubMed]

45. Watts, T.H.; Bertram, E.M.; Bukczynski, J.; Wen, T. T Cell Costimulatory Molecules in Anti-Viral Immunity: Potential Role in Immunotherapeutic Vaccines. Can. J. Infect. Dis. 2003, 14, 221-229. [CrossRef] [PubMed]

46. Heise, C.; Hermiston, T.; Johnson, L.; Brooks, G.; Sampson-Johannes, A.; Williams, A.; Hawkins, L.; Kirn, D. An adenovirus E1A mutant that demonstrates potent and selective systemic anti-tumoral efficacy. Nat. Med. 2000, 6, 1134-1139. [CrossRef]

47. Murakami, M.; Ugai, H.; Belousova, N.; Pereboev, A.; Dent, P.; Fisher, P.B.; Everts, M.; Curiel, D.T. Chimeric adenoviral vectors incorporating a fiber of human adenovirus 3 efficiently mediate gene transfer into prostate cancer cells. Prostate 2010, 70, 362-376. [CrossRef]

48. Kim, K.H.; Ryan, M.J.; Estep, J.E.; Miniard, B.M.; Rudge, T.L.; Peggins, J.O.; Broadt, T.L.; Wang, M.; Preuss, M.A.; Siegal, G.P.; et al. A new generation of serotype chimeric infectivity-enhanced conditionally replicative adenovirals: The safety profile of ad5/3-Delta24 in advance of a phase I clinical trial in ovarian cancer patients. Hum. Gene Ther. 2011, 22, 821-828. [CrossRef]

49. Kuryk, L.; Moller, A.W. Chimeric oncolytic Ad5/3 virus replicates and lyses ovarian cancer cells through desmoglein-2 cell entry receptor. J. Med. Virol. 2020, 92, 1309-1315. [CrossRef]

50. Kuryk, L.; Haavisto, E.; Garofalo, M.; Capasso, C.; Hirvinen, M.; Pesonen, S.; Ranki, T.; Vassilev, L.; Cerullo, V. Synergistic anti-tumor efficacy of immunogenic adenovirus ONCOS-102 (Ad5/3-D24-GM-CSF) and standard of care chemotherapy in preclinical mesothelioma model. Int. J. Cancer 2016, 139, 1883-1893. [CrossRef]

51. Kroemer, G.; Galluzzi, L.; Kepp, O.; Zitvogel, L. Immunogenic cell death in cancer therapy. Annu. Rev. Immunol. $2013,31,51-72$. [CrossRef]

52. Green, D.R.; Ferguson, T.; Zitvogel, L.; Kroemer, G. Immunogenic and tolerogenic cell death. Nat. Rev. Immunol. 2009, 9, 353-363. [CrossRef] 
53. McKenna, M.K.; Rosewell-Shaw, A.; Suzuki, M. Modeling the Efficacy of Oncolytic Adenoviruses In Vitro and In Vivo: Current and Future Perspectives. Cancers 2020, 12, 619. [CrossRef]

54. Olson, B.; Li, Y.; Lin, Y.; Liu, E.T.; Patnaik, A. Mouse Models for Cancer Immunotherapy Research. Cancer Discov. 2018, 8, 1358-1365. [CrossRef]

55. Kuryk, L.; Moller, A.W.; Garofalo, M.; Cerullo, V.; Pesonen, S.; Alemany, R.; Jaderberg, M. Antitumor-specific T-cell responses induced by oncolytic adenovirus ONCOS-102 (AdV5/3-D24-GM-CSF) in peritoneal mesothelioma mouse model. J. Med. Virol. 2018, 90, 1669-1673. [CrossRef]

56. Kuryk, L.; Haavisto, E.; Garofalo, M.; Capasso, C.; Hirvinen, M.; Pesonen, S.; Ranki, T.; Vassilev, L.; Cerullo, V. Synergistic Anti-Tumor Efficacy of Immunogenic Adenovirus ONCOS-102 and Standard of Care Chemotherapy in Preclinical Mesothelioma Model. Mol. Ther. 2016, 24, S262. [CrossRef]

57. Ranki, T.; Pesonen, S.; Hemminki, A.; Partanen, K.; Kairemo, K.; Alanko, T.; Lundin, J.; Linder, N.; Turkki, R.; Ristimaki, A.; et al. Phase I study with ONCOS-102 for the treatment of solid tumors-An evaluation of clinical response and exploratory analyses of immune markers. J. Immunother. Cancer 2016, 4, 17. [CrossRef]

58. American Cancer Society. Cancer Facts and Figures; American Cancer Society: Atlanta, GA, USA, 2018.

59. Ferlay, J.; Soerjomataram, I.; Ervik, M.; Dikshit, R.; Eser, S.; Mathers, C.; Rebelo, M.; Parkin, D.M.; Forman, D.; Bray, F. Globocan 2012: Estimated Cancer Incidence, Mortality and Prevalence Worldwide in 2012. Int. Agency Res. Cancer 2015, 136, E359-E386. [CrossRef]

60. Filley, A.C.; Dey, M. Immune System, Friend or Foe of Oncolytic Virotherapy? Front. Oncol. 2017, 7, 106. [CrossRef]

61. Jenkins, R.W.; Barbie, D.A.; Flaherty, K.T. Mechanisms of resistance to immune checkpoint inhibitors. Br. J. Cancer 2018, 118, 9-16. [CrossRef]

62. Sharma, P.; Hu-Lieskovan, S.; Wargo, J.A.; Ribas, A. Primary, Adaptive, and Acquired Resistance to Cancer Immunotherapy. Cell 2017, 168, 707-723. [CrossRef]

63. Lugowska, I.; Teterycz, P.; Rutkowski, P. Immunotherapy of melanoma. Contemp. Oncol. 2018, 22, 61-67. [CrossRef]

64. Eggermont, A.M.M.; Crittenden, M.; Wargo, J. Combination Immunotherapy Development in Melanoma. Am. Soc. Clin. Oncol. Educ. Book 2018, 38, 197-207. [CrossRef]

65. Michielin, O.; Atkins, M.B.; Koon, H.B.; Dummer, R.; Ascierto, P.A. Evolving impact of long-term survival results on metastatic melanoma treatment. J. Immunother. Cancer 2020, 8, e000948. [CrossRef] [PubMed]

66. Smalley, K.S.; Eroglu, Z.; Sondak, V.K. Combination Therapies for Melanoma: A New Standard of Care? Am. J. Clin. Derm. 2016, 17, 99-105. [CrossRef] [PubMed]

67. Yu, C.; Liu, X.; Yang, J.; Zhang, M.; Jin, H.; Ma, X.; Shi, H. Combination of Immunotherapy With Targeted Therapy: Theory and Practice in Metastatic Melanoma. Front. Immunol. 2019, 10, 990. [CrossRef] [PubMed]

68. Dharmadhikari, N.; Mehnert, J.M.; Kaufman, H.L. Oncolytic virus immunotherapy for melanoma. Curr. Treat. Options Oncol. 2015, 16, 326. [CrossRef] [PubMed]

69. Kuryk, L.; Bertinato, L.; Staniszewska, M.; Pancer, K.; Wieczorek, M.; Salmaso, S.; Caliceti, P.; Garofalo, M. From Conventional Therapies to Immunotherapy: Melanoma Treatment in Review. Cancers 2020, 12, 3057. [CrossRef] [PubMed]

70. Peter, M.; Kuhnel, F. Oncolytic Adenovirus in Cancer Immunotherapy. Cancers 2020, 12, 3354. [CrossRef] [PubMed]

71. Oh, C.M.; Chon, H.J.; Kim, C. Combination Immunotherapy Using Oncolytic Virus for the Treatment of Advanced Solid Tumors. Int. J. Mol. Sci. 2020, 21, 7743. [CrossRef]

72. Merlino, G.; Herlyn, M.; Fisher, D.E.; Bastian, B.C.; Flaherty, K.T.; Davies, M.A.; Wargo, J.A.; Curiel-Lewandrowski, C.; Weber, M.J.; Leachman, S.A.; et al. The state of melanoma: Challenges and opportunities. Pigment. Cell Melanoma Res. 2016, $29,404-416$. [CrossRef]

73. Rughani, M.G.; Gupta, A.; Middleton, M.R. New treatment approaches in melanoma: Current research and clinical prospects. Adv. Med. Oncol. 2013, 5, 73-80. [CrossRef]

74. Chu, R.L.; Post, D.E.; Khuri, F.R.; Van Meir, E.G. Use of replicating oncolytic adenoviruses in combination therapy for cancer. Clin. Cancer Res. 2004, 10, 5299-5312. [CrossRef]

75. Goradel, N.H.; Mohajel, N.; Malekshahi, Z.V.; Jahangiri, S.; Najafi, M.; Farhood, B.; Mortezaee, K.; Negahdari, B.; Arashkia, A. Oncolytic adenovirus: A tool for cancer therapy in combination with other therapeutic approaches. J. Cell Physiol. 2019, 234, 8636-8646. [CrossRef]

76. Kuryk, L.; Moller, A.-S.W.; Jaderberg, M. The Combinatory Treatment of the Oncolytic Adenovirus ONCOS-102 with Anti PD-1 (Keytruda (R)) Show Synergistic Anti-Tumor Effect in Humanized A2058 Melanoma huNOG Mouse Model. Mol. Ther. 2018, 26, 200-201.

77. Chaurasiya, S.; Fong, Y.; Warner, S.G. Optimizing Oncolytic Viral Design to Enhance Antitumor Efficacy: Progress and Challenges. Cancers 2020, 12, 1699. [CrossRef]

78. Beatty, M.S.; Curiel, D.T. Chapter two-Adenovirus strategies for tissue-specific targeting. Adv. Cancer Res. 2012, 115, 39-67. [CrossRef]

79. Hensen, L.C.M.; Hoeben, R.C.; Bots, S.T.F. Adenovirus Receptor Expression in Cancer and Its Multifaceted Role in Oncolytic Adenovirus Therapy. Int. J. Mol. Sci. 2020, 21, 6828. [CrossRef] 
80. Kuryk, L.; Moller, A.W.; Jaderberg, M. Combination of immunogenic oncolytic adenovirus ONCOS-102 with anti-PD-1 pembrolizumab exhibits synergistic antitumor effect in humanized A2058 melanoma huNOG mouse model. Oncoimmunology 2019, 8, e1532763. [CrossRef]

81. Marchini, A.; Daeffler, L.; Pozdeev, V.I.; Angelova, A.; Rommelaere, J. Immune Conversion of Tumor Microenvironment by Oncolytic Viruses: The Protoparvovirus H-1PV Case Study. Front. Immunol. 2019, 10, 1848. [CrossRef]

82. Zhang, Y.; Zhang, Z. The history and advances in cancer immunotherapy: Understanding the characteristics of tumor-infiltrating immune cells and their therapeutic implications. Cell Mol. Immunol. 2020, 17, 807-821. [CrossRef]

83. Saito, R.; Kobayashi, T.; Kashima, S.; Matsumoto, K.; Ogawa, O. Faithful preclinical mouse models for better translation to bedside in the field of immuno-oncology. Int. J. Clin. Oncol. 2020, 25, 831-841. [CrossRef]

84. Models for Immuno-oncology Research. Cancer Cell 2020, 38, 145-147. [CrossRef] [PubMed]

85. Robinson, M.; Li, B.; Ge, Y.; Ko, D.; Yendluri, S.; Harding, T.; VanRoey, M.; Spindler, K.R.; Jooss, K. Novel immunocompetent murine tumor model for evaluation of conditionally replication-competent (oncolytic) murine adenoviral vectors. J. Virol. 2009, 83, 3450-3462. [CrossRef] [PubMed]

86. Halldén, G.; Hill, R.; Wang, Y.; Anand, A.; Liu, T.-C.; Lemoine, N.R.; Francis, J.; Hawkins, L.; Kirn, D. Novel immunocompetent murine tumor models for the assessment of replication-competent oncolytic adenovirus efficacy. Mol. Ther. 2003, 8, 412-424. [CrossRef]

87. Jogler, C.; Hoffmann, D.; Theegarten, D.; Grunwald, T.; Uberla, K.; Wildner, O. Replication properties of human adenovirus in vivo and in cultures of primary cells from different animal species. J. Virol. 2006, 80, 3549-3558. [CrossRef]

88. Bramante, S.; Kaufmann, J.K.; Veckman, V.; Liikanen, I.; Nettelbeck, D.M.; Hemminki, O.; Vassilev, L.; Cerullo, V.; Oksanen, M.; Heiskanen, R.; et al. Treatment of melanoma with a serotype 5/3 chimeric oncolytic adenovirus coding for GM-CSF: Results in vitro, in rodents and in humans. Int. J. Cancer 2015, 137, 1775-1783. [CrossRef]

89. Targovax Announces Impressive Objective Responses as Well as Effects on Non-Injected Lesions in ONCOS-102 Trial in Anti-PD1 Refractory Melanoma Patients. Available online: https://www.targovax.com/en/targovax-announces-impressive-objectiveresponses-as-well-as-effects-on-non-injected-lesions-in-oncos-102-trial-in-anti-pd1-refractory-melanoma-patients / (accessed on 26 January 2021).

90. Frohlich, A.; Hoffmann, F.; Niebel, D.; Egger, E.; Kukuk, G.M.; Toma, M.; Sirokay, J.; Bieber, T.; Landsberg, J. Talimogene Laherparepvec in Advanced Mucosal Melanoma of the Urethra Upon Primary Resistance on Immune Checkpoint Inhibition: A Case Report. Front. Oncol. 2020, 10, 611. [CrossRef]

91. Hamid, O.; Ismail, R.; Puzanov, I. Intratumoral Immunotherapy-Update 2019. Oncologist 2020, 25, e423-e438. [CrossRef] 